\title{
NARROW RESONANCES IN STATISTICAL MECHANICS
}

$R, F$, Dashen and $R$, Rajaraman

The Institute for Advanced Study, Princeton, New Jersey 08540

\section{ABSTRACT}

It is shown that when the dynamics of a systern is dominated by narrow resonance formation it behayes like a non-interacting system with added species of free particles, corresponding to all the quantum numbers and the statistics of each of the resonances. This equivalence, widely assumed and used in practice, is established by explicitly constructing many particle $S$ matrices consistent with unitarity, that correspond to purely resonance dominated dynamics. Thẹge are then used in the S matrix formulation of ptatistical mechanics to obtain the grand partition function, which is seen to reveal the above result. In the following paper, operational criteria are presented for determining when in general narrow resonances and loosely bound states may be treated as "elementary" particles.

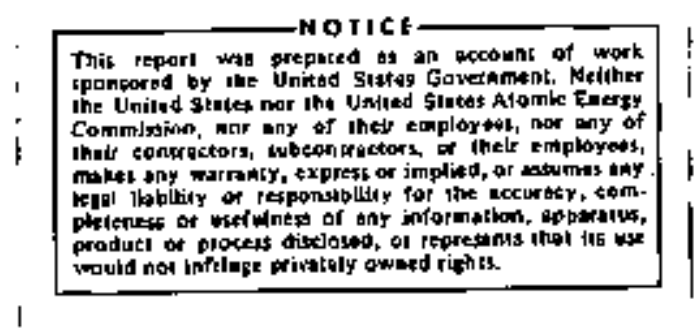

$\uparrow$

On leave from the University of Delhi, Delhi 7, India. Research sponsored by the Atomic Energy Commission, Grant No. AT (11-1) $=2220$. 


\section{DISCLAIMER}

This report was prepared as an account of work sponsored by an agency of the United States Government. Neither the United States Government nor any agency Thereot, nor any of their employees, makes any warranty, express or implied, or assumes any legal liability or responsibility for the accuracy, completeness, or usefulness of any information, apparatus, product, or process disclosed, or represents that its use would not infringe privately owned rights. Reference herein to any specific commercial product, process, or service by trade name, trademark, manufacturer, or otherwise does not necessarily constitute or imply its endorsement, recommendation, or favoring by the United States Government or any agency thereof. The views and opinions of authors expressed herein do not necessarlly state or reflect those of the United States Government or any agency thereof. 


\section{DISCLAIMER}

Portions of this document may be illegible in electronic image products. Images are produced from the best available original document. 


\section{INTRODUCTION}

A popular notion, used in a variety of physical situations, asserts that narrow resonances may be treated to a good approximation on the same footing as stable elementary particles. We are quite accustomed to the idea of treating neutrons, pions, muons, etc., all of which are actually unstable, as if they were "elementary." It is assumed that in'a collection of such objets, each species would obey its own separate statistics, and that one can ignore any constraints of symmetrization or antisymmetrization between wave functions of, say, a neutron and an electron. A possibly more debatable example of stach use arises in certain calculations of the equation of state of neutron star interiors. Here, not only the neutrons, the $\Lambda_{r}$ and the $\Sigma$, but often also the 3-3 resonance $(\Delta)$ is treated as a separate species. This as sumption allows one to put, for instance, $a \Delta$ and a nucleon in the same momentum state, and leads to a great saving in kinetic energy at high baryon densities.

Yet, all these anstable particles are also expected to occur as resonances in the scattering of stable particles. This is certainly true of the hadronic resonance $\Delta$, which occurs in the $N \pi$ channel, and presumably pions, muons, neutrons, etc. would also occur as resonances in the appropriate weak interaction scattering experiments, if one could perform them. Thus, at least in part, the resonances may be composite objects. If so, one would expect some constraint between their wavefunctions, and those of the "constituent" stable particles. 
The difficulty is that we do not know at present, to what extent these resonances are really composite or elementary. It would therefore be nice to determine criteria under which narrow.resonances may be considered approximately as elementary, even if in fact they were fully composite. Further these criteria, assuming one can find them, shotuld be specified operationally, not involving ultimate knowlcdge of whetler a resonance is truly composite, elementary, or both, in any absolute sense. Finally, when a resonance may be treated as a separate elementary species, one should be able to justify the neglect of possible symmetriation constraints with its "constituent" stable particles.

We attempt, in this paper and the one following it, to throw some light on these issues, along with the corresponding problems for loosely bound composite systems.

We begin, in Section II by considering a collection of two stable species, taking for generality one as a fermion and the other a boson. We assume that the two species interact only to the extent of forming a narrow resonance. This specification is made precise by giving a unitarily consistent $S$ matrix for the scattering of $m$ fermions with $n$ bosons for all $\{n, m\}$. Then, waing the S-matrix formulation of Statistical Mechanics, ${ }^{1}$ we calculate the Grand Partition Function $Z$ for this interacting system at any temperature. The reas on behind evaluating $Z$ is that apart from giving all other thermodynamic functions, it also resolves questions of relative statistics. Since all many-particle Smatrices are seen to exist even when the dynamics consists only of a narrow Iesonance, all vìial coefficients also exist. However, in the limit of a 
narrow resonance; these virial coefficients can be summed. The resulting contribution to the partition function is shown to be exactly what a third ideal ga $\mathrm{a}$ would give, if it had Fermi statistics and the mass and quantum numbers of the regonance. Note that for Boltzmann gases, such a proof would be trivial, since only the second virial coefficient exists for a purely narrow resonant scattering. This coefficient, given by the Beth-Uhlenbeck formula ${ }^{2}$ in terms of the elastic phase shift, immediately reduces to an "ideal" Boltzmann gas term corresponding to the resonance. However, the questions we posed earlier are meaningful only for (the more realistic) Bose and Fermi.syztems, in which case the caleulation involves all virial coefficientis and is more complicated.

In Section III we extend this result to cases where such narrow resonances "scatter" with other narrow resonances or stable particles. Of particular interest is the case where the scattering of narrow resonances with one another and with stable particles, is again dominated by narrow resonance formation. Such dynamics, dominated by tree graphs involving a heirarchy of narrow resonances, is used in both the dual models ${ }^{3}$ and the thermodyoamic theories of elementary particles. ${ }^{4}$ We find that such dynamics can once again be replaced by a sum of ideal gas terms, one for each resonance.

Reconciliation of these results with the need to symmetrize for antisymmetrize) the "constituents" of the resonance with other stable particles involves the use of Levinson's Theorem. The same theorem also throws light on the status of "loosely" bound states. By "loosely bound" states, we mean states bound by potentials whose strength and inverse range are small compared to the temperature and the density. These and related topics form the content of the 
succeeding paper. It has been presented separately in order not to drown the physics there amongst the technical details of narrow resonance algebra in this paper.

I. TWO SPECIES INTERACTING THROUGH A NARROW RESONANCE

We will calculate the exact grand partition function, from which other thermodynamic functions can be derived, for the following system:

There are two stable species of particles, say, one a boson and the other a fermion, each carrying a separately conserved quantum number. To facilitate discussion, let us call the boson a $\pi^{*}$, carrying charge and the fermion as a neutron (n), carrying baryon number. Both particles are stable in the absence of weak interactions. These specifications are just for conve-. nience. Our results are adaptable to cases of two fermions, or two bosons; whether or not they carry separate quantum numbers.

Let us assume that there are no $\pi^{-}-\pi^{-}$interactions and no $n=n$ interactions. Further, let the $\pi^{-}-\mathrm{n}$ interaction be such as to produce only a narrow s-wave resonance (called the $N^{*}$ ) in the elastic $\pi^{-}$n scattering amplitude, both on and off the energy shell. That is, let (see Fig. la)

$$
\left\langle p^{\prime} k^{\prime}|T(E)| p, k\right\rangle=\frac{\left\{(\pi)^{3} g^{2} \delta^{3}\left(\vec{p}+\vec{k}-\overrightarrow{p^{\prime}}-\overrightarrow{k^{\prime}}\right)\right.}{E^{\prime}-\sqrt{M^{2}+(\vec{p}+\vec{k})^{2}}+i \Gamma}
$$

and the S-matrix

$$
\left\langle p^{\prime} k^{\prime}|s| p k\right\rangle=\left\langle p^{l} k^{\prime}\left|1-2 \pi i \delta\left(e+\omega-e^{t}-\omega^{\prime}\right) T(e+\omega)\right| p k\right\rangle
$$


where

$$
\begin{aligned}
M & =\text { mass of the resonance } N^{*} \\
2 \Gamma & =(2 \pi)^{4} g^{2} \sum_{p, k} \delta^{3}(\bar{p}+\vec{k}) \delta(e+\omega-M)
\end{aligned}
$$

and we will always use the combinations $p_{i}=\left(\bar{p}_{i}, e_{i}\right)$ and $k_{i}=\left(\vec{k}_{i}, \omega_{i}\right)$ for the four momenta of neutrons and pions respectively.

We will work in the narrow resonance limit, i. e., $g^{2} \rightarrow 0$, when the width $\Gamma$ also goes to zero and the amplitude in eq. (2.1) is unitary. Also, since the interaction in (2.1) is spin independent, the "neutron" is effectively spinless in our problem.

Turning to the many particle T-matrix, we make a corresponding assumption consistent with eq, 2.1 for the $\pi^{-} \mathrm{n}$ amplitude. We as sume that it consists of and only of diagrams, where real (or virtual) pions repeatedly form narrow elastic $N^{*}$ resonances with other real (or virtual) neutrons. Unitarity forbids as from using a two particle amplitude of eq. (2.1) without. permitting many particle amplitudes as in Fig. 2a. Thus the T-matrix for the scattering of 2 pions and 3 nucleons would be the sum of all terms of the type shown in Fig. 2a. [Note that several disconnected pieces like this are also permitted. However, a diagram like Fig. $2 b$ is not permitted since two successive resonances occur between the same $\pi$ "n pair. Since the resonance in eg. 2. 1 is already unitary in two particle space, Fig. 2b would lead to double counting.]

Diagrams such as Fig. 2a stand here for terms in a Lippmann Schwinger expansion of the $T(E)$ matrix. To be precise, a diagram like Fig. 3 stands for the expression 
Fig. $3=\frac{(2 \pi)^{3} g^{2} \delta^{3}\left(\overrightarrow{p_{1}}+\overrightarrow{k^{i}}-\vec{k}_{5}-\vec{p}_{3}^{i}\right)}{E-s_{2}-\sqrt{M^{2}+\left(\vec{p}_{1}+\vec{k}^{2}\right.}+i \Gamma} \frac{1}{E-\omega_{5}-e_{3}-e_{2}+i t}$
$\frac{(2 \pi)^{3} g^{2} \delta^{3}\left(\vec{k}_{5}+\vec{p}_{2}-\vec{k}_{4}-\vec{p}_{2}^{\prime}\right)}{E-e_{3}-\sqrt{M^{2}+\left(\vec{k}_{5}+\vec{p}_{2}\right)^{2}}+i \Gamma} \frac{1}{E-\omega_{4}-e_{3}-e_{2}^{1}+i \epsilon} \frac{(2 \pi)^{3} g^{2} \delta^{3}\left(\vec{k}^{t}+\vec{p}_{1}^{\prime}-\vec{k}_{4}-\vec{p}_{3}\right)}{E-e_{2}^{t}-\sqrt{M^{2}+\left(\vec{p}_{3}+\vec{k}_{4}\right)^{2}}+i \Gamma}$

From this illustration, the corcoponding expression for any diagram for the many particle $T(E)$ can be written down, and the assumed dynamics of one narrow resonance gystem is fully specified.

With this information, the full Grand Partition Function $Z$ can in principle be evaluated using the S-matrix formulation of Statistical Mechanics. For our system,

$$
\begin{aligned}
\frac{p V}{\mathbf{k T}}=\log Z & =\log z_{0}+ \\
& \frac{1}{2 \pi i} \sum_{n_{1} n_{2}} e^{\beta\left(\mu_{1} n_{1}+\mu_{2} n_{2}\right)} \int d E e^{-\beta E}\left[\operatorname{Tr} n_{1} n_{2} A S^{-1}(E) \frac{\partial}{\partial E} S(E)\right]_{c}
\end{aligned}
$$

where $S(E)=1-2 \pi i \delta\left(E-H_{0}\right) T(E)$

$\mathrm{H}_{0} \quad=$ free Hamiltonian

$\mu_{1}=$ chemical potential for the $\pi^{-}$, corresponding to charge conservation

$\mu_{2}=$ chemical potential for the neutron, for baryon conservation

A = Boson symmetrization and Fermion antisymmetrization operator $n_{1}\left(n_{2}\right)$ is the number of pions (nucleons) in a state, and the subscript c stands for the connected part of the combination. $\operatorname{Tr}\left[\mathrm{AS}^{-1} \frac{\partial}{\partial E} \mathrm{~S}\right]$. Note 
that two apparently disconnected pieces can be "connected" through their final state labels, thanks to the exchange operator $A^{1} z_{0}$ in eq. 2.5 is the noninteracting value of $Z$ for the two species, viz.,

$$
\begin{aligned}
\log z_{0}=v \int & \frac{a^{3} p}{(2 \pi)^{3}} \log \left[1+e^{-\beta\left(\sqrt{P^{2}+m_{n}^{2}}-\mu_{2}\right)}\right] \\
& -v \int \frac{d^{3} k}{(2 \pi)^{3}} \log \left[1-e^{-\beta\left(\sqrt{\left.k^{2}+m_{n}^{2}-\mu_{1}\right)}\right]}\right.
\end{aligned}
$$

Given an S-matrix tor $n_{1}$ pions and $n_{2}$ nucleons as specified for all $n_{1}$ and $n_{2}$, all virial coefficients in the double expansion in (2.5) exist. In the limit $g^{2}-0$, we show that these can all be evaluated and summed.

[Incidentally, we have added an Appendix at_the end of the following paper, clarifying what the $\frac{\partial}{\partial E}$ means, given a general S-matrix element as a function of several energy variables. In this paper, we have postulated S-matrix elements with explicit off energy shell (E) dependence, so that $\frac{\partial}{\partial E}$ is unambiguous. ]

To begin, note that every contribution to any many particle T-matrix is, apart from momentum conserving $\delta$-functions, a product of free Green's functions $\frac{1}{E \sim H_{0}+i \epsilon}$, and "resonance propagators" of the form $\frac{g^{2}}{x_{i}+i \Gamma}$ where $x_{i}$ are some energy variables made up of $E$, the resonance mass $M$ and the energy-momenta of other particles in the diagram. (See for example eq. 2.4). Remember that $\Gamma_{\propto \mathrm{g}^{2}}$ from $(2.3)$, and that

$$
\begin{aligned}
& \lim _{\epsilon \rightarrow 0}\left(\frac{\epsilon}{x+i \epsilon}\right)^{n}=0=\frac{1}{x+i \epsilon}\left(\frac{\epsilon}{x+i \epsilon}\right)^{n} \\
& \lim _{\epsilon \rightarrow 0}\left(\frac{\epsilon}{x-i \epsilon}\right)^{m}\left(\frac{\epsilon}{x+i \epsilon}\right)^{n}=0
\end{aligned}
$$


while

$$
\lim _{\epsilon \rightarrow 0}\left(\frac{2 \xi}{x-i \epsilon}\right)^{n} \frac{\partial}{\partial x}\left(\frac{2 \epsilon}{x+i \epsilon}\right)^{m}=\frac{2 \pi i}{(i)^{m+n}}(-1)^{n} \frac{(n+m-1) !}{(n-1) !(m-1) !} \delta(x)
$$

for all integral $n_{t} m>0$.

From this one can see that all the $T$ matrices vanish as $g^{2}-0$, since they all have $g^{2}$ dependence of the form $\pi \frac{g^{2}}{x_{i}+i \vec{\Gamma}}$. So does $\frac{a}{\partial E} T(E)$ and, for that matter, $\mathrm{T}^{+} \mathrm{T}$. However, the combination $\ddot{T}^{+} \frac{\partial}{\partial \mathrm{E}} \mathrm{T}$ from eq. 2.5 can survive since poles from $x=$ if and $x=-$ is can pincli the real axis for some energy variable $x$, giving a $6(x)$ as per (2, 8). Note while using (2. 5), that in the combination $T^{+}(E) \frac{\partial}{\partial E}\left[\delta\left(E-H_{0}\right) T(E)\right]$, when $\frac{\partial}{\partial E}$ acts on the $\delta\left(E-H_{0}\right)$, that is to say, on $n$ particle phase space $\rho_{n}(E)$, giving $\mathrm{T}^{+}(\mathrm{E}) \mathrm{T}(\mathrm{E}) \frac{\partial}{\partial E} P_{n}(\mathrm{E})$, the tegult is again zero as $\mathrm{g}^{2}-0$, due to (2. 7b). Thus, eq. 2.5 reduces to

$\log Z=\log Z_{0}+\frac{(2 \pi)^{2}}{2 \pi i} \sum_{n_{1}, n_{2}} e^{\beta\left(\mu_{1} n_{1}+\mu_{2} n_{2}\right)} \int d E e^{-\beta E}\left[T_{\text {race }} A \delta\left(E-H_{0}\right) T^{+}(E) \delta\left(E-H_{0}\right) \frac{\partial}{\partial E} T(E)\right]_{C}$ $=\log Z_{0}+\sum_{n_{1} n_{2}} e^{\beta\left(\mu_{1} n_{1}+\mu_{2} n_{2}\right)} \quad b\left(n_{1} n_{2}\right)$

The physics of the singular nature of such a narrow resonance can be understood better by looking at the lowest virial term in the expansion (2.9), corresponding to $n_{1}=n_{2}=1$. It involves the elastic $\pi^{-} n$ amplitude given in $(2,1)$, which as $g^{2}-0$ vanishes everywhere in $E$, except at the resonance energy, where it is finite. The same is true of the cross section, related to $|T(E)|^{2}$, which also has a spike of finite height and decreasing width as $\mathrm{g}^{2}-0$. Neither the cross section, nor the amplitude, if integrated over the energy will contribute any* thing. However the partition function is an integral over $S^{-1} \frac{\partial}{\partial E} S$, which 
may be interpreted as the cross section multiplied by the "time delay" due to interaction. For a narrow resonance, this "time delay" clearly becomes infinite. As a result, $s^{-1} \frac{\partial}{\partial E} s$ develop enough strength at the resonance point to behave like a $\delta$-function in energy. This is seen when $b(1,1)$ is obtained by substituting $(2,1)$ into $(2,9)$. We get

$b(1,1)=\frac{\delta^{3}(0)}{2 \pi i} \int d^{3} P \int d E e^{-\beta E}[2 \Gamma(E, P)]^{2} \frac{1}{E-\sqrt{M^{2}+P^{2}}-i \Gamma} \frac{\theta}{\partial E} \frac{1}{E-\sqrt{M^{2}+P^{2}}+i \Gamma}$ where, analogous to the definition of the width $\Gamma$ in eq. (2.3), we define

$$
2 \Gamma(E, P)=g^{2}(2 \pi)^{4} \sum_{k, P} \delta^{3}(\vec{k}+\vec{p}-\bar{P}) \delta(e+\omega-E)
$$

Then as $g^{2}$ (and hence $\Gamma$ and $\Gamma(E, P)$ ) goto zero, using (2.8),

$$
\begin{aligned}
b(1,1) & =\delta^{3}(0) \int d^{3} P \int d E e^{-\beta E}\left(\frac{r(E, P)}{\Gamma}\right)^{2} \delta\left(E-\sqrt{M^{2}+P^{2}}\right) \\
& =V \int \frac{d^{3} P}{(2 \pi)^{3}} e^{-\beta \sqrt{M^{2}+F^{2}}}
\end{aligned}
$$

The result (2.10), where the resonance behaves just like another free particle of masa $M$ is very familiar. It is derived in ref. (2) and. is also easily available from the old Beth-Uhlenbeck formula. ${ }^{2}$ For a Boltzmann gas, this is the only contribution in the narrow resonance limit to the whole virial series, and is the basis for the notion that natrow resonances behave like stable particles. For a realistic Bose or Fermi gas, all the higher virial coefficients in the series (2.9) also exist and must be calculated and summed. This is really the only new element in our work in this section. As hoped for, these higher virial contributions merely provide the " $\mathrm{N}^{*}$ species" with the appropriate (in this example, Fermi Dirac) statistics. But this simple result evolves 
only after some interesting cancellations between classes of diagrams, which amount to a self consistent change in the resonance width in the presence of the medium.

To evaluate the higher virial coefficients, we again rely on the identities (2.7) and (2,8). Every n-particle T matrix diagram, when put on the energy ehell (as required by the $6\left(\mathrm{E}-\mathrm{H}_{0}\right\}$ in 2.9$)$, involves a product of resonance propagators $\pi_{i} \frac{g^{2}}{x_{i}+i \Gamma} \cdot$ Each $x_{i}$ is just $E_{i}-E_{i}^{(r)}$, where $E_{i}$ is the energy of the ${ }^{-}$n pair forming that resonance and $\mathbf{E}^{r}$ is the resonance energy for that particular total momentum. The combination $\mathrm{T}^{+} \frac{\partial}{\partial \mathrm{E}} \mathrm{T}$ will then be of the form

$$
\left\{\prod_{i}\left(\frac{\dot{s}^{2}}{x_{i}-i \Gamma}\right)\right\}\left\{\prod_{j}\left(\frac{g^{2}}{x_{j}+i \Gamma}\right)\right\}\left\{\sum_{j}\left(\frac{-1}{x_{j}+i \Gamma}\right)\right\}
$$

Note that the sum over state labels (phase space integrals) can be written as integrals over independent pair energies $x_{i}$ and any remaining variables, with a suitable Jacobian. Apart from the resonance propagators, $T^{+} \frac{\partial}{g E} T$ involves momentum conserving $\delta$ functions, energy canserving $\delta$ functions, and Green's functions $\frac{1}{E-H_{0}-i a}$ whose imaginary parts again conserve appropriate cnergies.

As a result of these $\delta$ functions, two things may happen to any given $T^{+} \frac{\partial}{\partial E} T$ contribution:

(a) All the energy variables $x_{i}$ and $x_{j}$ in $\{2.11)$ get constrained to be the same. In that case (2. 11) has the form $\left(\frac{g^{2}}{x_{i}-i \Gamma}\right)^{m} \frac{\partial}{\partial x_{i}}\left(\frac{g^{2}}{x_{i}+i \bar{\Gamma}}\right)^{n}$, which by (2.8) survives on integration over $x_{i}$. Such a diagram will thus survive. (b) At least two independent $x^{\prime} s$ remain in (2.11). In that case, in the exparsion in $(2,11)$ at least one of them $\left(x_{1}\right)$ will involve $\left(\frac{g^{2}}{x_{1}-i \Gamma}\right)^{m}$ or $\left(\frac{g^{2}}{x_{1}+i \Gamma}\right)^{n}$ 
or $\left(\frac{g^{2}}{x_{1}-i \Gamma}\right)^{m}\left(\frac{g^{2}}{x_{1}+i \Gamma}\right)^{n}$, any of which make the integral over $x_{1}$ and hence the contribution of the entire diagram vanish.

The interested reader can verify this argument by considering some examples. A diagram such a $\mathrm{Fig}$. 3, when inserted either for $\mathrm{T}^{+}$or for $T$ in $T^{+} \frac{\partial}{\partial E} T$ will destroy that contribution. On the other hand, the lowest virial coefficient $b(1,1)$ survived since essanitially only one energy resonated (Fig. lb), for a given total momentum in both $T^{+}(E)$ and $T(E)$.

The criterion then is that only those contributions to the $n$ particle

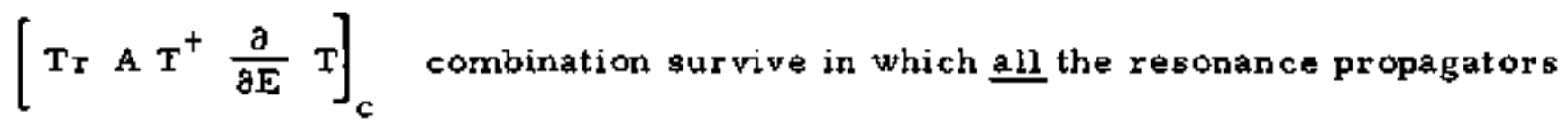
are constrained by energy momentum conservation to be the game. This important technical criterion, along with identities (2.7) and (2.8), will be used repeatedly.

Before we collect all the terms that satisfy the criterion, and survive, let us start with a simple example that does, (Fig. 4a). It's contribution to $\log z$ in eq. (2.9) is.

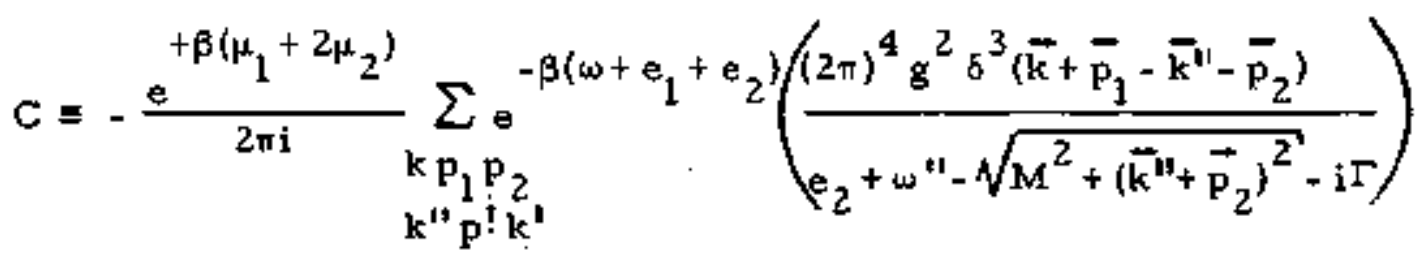

$$
\begin{aligned}
& \frac{1}{e_{1}+\omega+e_{2}-\left(e_{2}+e_{2}-w^{\prime \prime}\right)-i c}\left(\frac{g^{2}(2 \pi)^{3} \delta^{3}\left(\overrightarrow{p_{2}}+\overrightarrow{k^{\prime \prime}}-\overrightarrow{p^{\prime}}-\overrightarrow{k^{\prime}}\right)}{e^{\prime}+\omega^{\prime}-\sqrt{M^{2}+\left(\overrightarrow{\left.p^{\prime}+\vec{k}^{\prime}\right)^{2}}-i \Gamma\right.}}\right) \\
& \text { - } 2 \pi \delta\left(e^{\prime}+\omega^{\prime}+e_{2}-e_{1}-e_{2}-\omega\right)\left(\frac{(2 \pi)^{3} g^{2} \delta^{3}\left(\overline{p_{1}}+\bar{k}-\bar{p}^{\prime}-\overline{k^{\prime}}\right)(-1)}{\left(e_{1}+\omega-\sqrt{M^{2}+\left(\overrightarrow{p_{1}}+\vec{k}\right)^{2}}+i \Gamma\right)^{2}}\right)
\end{aligned}
$$


The minus sign arises because the $\mathrm{p}_{1} \mathrm{p}_{2}$ lines have been exchanged. Retain only the $+i \pi \delta\left(e_{1}+\omega-e_{2}-\omega^{\prime l}\right)$ in the Green's function in $(2.12\}$. We will come to the principal part in a moment. Note that because of momentum conserva tion, all resonance propagators are the same, Let $\vec{k}+\vec{p}_{1}=\bar{p}$ and $\omega+e_{1}=E$. Then the expression in (2.12) is merely

$C \pm \frac{\delta^{3}(0)}{2 \pi i} e^{+\beta\left(\mu_{1}+\mu_{2}\right)} \sum_{k, p_{1}} e^{-\beta\left(\omega+e_{1}\right)} \frac{(2 \pi)^{4} g^{2}}{\left(E-\sqrt{M^{2}+P^{2}}-i \Gamma\right)^{2}} \frac{-i \tilde{\gamma}_{N}(E, P) 2 \Gamma(E, P)}{\left(E-\sqrt{M^{2}+F^{2}}+i \Gamma\right)^{2}}$

where $2 \mathrm{r}(\mathrm{E}, \overrightarrow{\mathrm{P}})$ as before equals $\sum_{\mathrm{k}^{\prime} \mathrm{p}^{\prime}} \delta^{3}\left(\overrightarrow{\mathrm{k}}^{1}+\overrightarrow{\mathrm{p}}^{\prime}-\overline{\mathrm{P}}\right) \delta\left(\mathrm{e}^{\prime}+\omega^{\prime}-E\right)(2 \pi)^{4} \mathrm{~g}^{2}$ and

$$
2 \tilde{y}_{N}\left(E, \vec{P}, \mu_{2}\right)=\sum_{k^{\prime \prime} p_{2}} e^{-\beta\left(e_{2}-\mu_{2}\right)} \delta^{3}\left(\vec{k}^{\prime \prime}+\vec{p}_{2}-\bar{P}\right) \delta\left(\omega "+e_{2}-E\right)(2 \pi)^{4} g^{2}
$$

It is clear from the identity $(2,8)$ that as $g^{2} \rightarrow 0,(2,13)$ will give a term proportional to $\delta\left(E-\sqrt{P^{2}+M^{2}}\right.$, which will survive upon integration over $\mathbf{E}=\omega+\mathbf{e}$.

Next, we add to Fig. 4a, a sequence of terms (such as in Fig. 4 b) where an arbitrary number of non-interacting neutron lines are "exchanged"1 with the nucleon $\mathrm{P}_{Z}$. This will only replace in $(2,13)$, the factor $2 \tilde{Y}_{\mathrm{N}}$ by a factor

$$
2 \gamma_{N}\left(E, \vec{P}, \mu_{2}\right)=\sum_{k^{\prime \prime} p_{2}} \delta^{3}\left(\vec{k}^{\prime \prime}+\vec{p}_{2}-\vec{P}\right) \delta\left(\omega^{\prime \prime}+e_{2}-E\right) \frac{e^{-\beta\left(e_{2}-\mu_{2}\right)}(2 \pi)^{4} g^{2}}{1+e^{-\beta\left(e_{2}-j \mu_{2}\right)}}
$$

$$
2 Y_{N}\left(E, \bar{P}_{,} \mu_{2}\right) \text { is clearly just the "reduced" phase space for two }
$$

particles of energy-momentum $(E, \vec{P})$, weighted by the neutron occupation probability. 
Similarly, the diagrams depicted in Fig. 5 will give a term similar to (2.13) except that $-i \tilde{\gamma}_{N}$ will now be replaced by $+i \gamma_{\pi}\left(E, p, \mu_{1}\right)$ given by

$$
2 \gamma_{\pi}\left(E, \vec{p}, \mu_{1}\right)=\sum_{k^{\prime \prime} p_{2}} \delta^{3}\left(\vec{k} "+\vec{p}_{2}+F\right) \delta\left(\omega^{\prime \prime}+e_{2}-E\right) \frac{e^{-\beta\left(\omega^{\prime \prime}-\mu_{1}\right)}}{1-e^{-\beta\left(\omega^{\prime \prime}-\mu_{1}\right)}(2 \pi)^{4}} g^{2}
$$

Racher than evaluate these contributions separately, we now identify a whole sequence of such diagrams, which effectively involve only one resonance denominator. These diagrams are generated as follows, Consider Fig. 6, in which the shaded blob on the right-hand side is iterated tu give a sequence of $T$ matrix diagrams. As illustrated in eq. $(2,4)$ for the case of Fig, 3 , the precise algebraic expression represented by any $T$ matrix diagram is defined. Now insert the shaded blob in Fig, 6, into all the $T^{+} \frac{\partial}{\partial E} T$ combinations depicted in Fig. 7. In both Figures 6 and 7 , the non-interacting pion for neutron) lines are meant to be arbitrary in number, beginning with zero, and are exchanged with the $k_{1}$ (or $p_{1}$ ) line. Their effect is merely to introduce the Bose (or Fermi) occupation probability in the phase space integral of the $k_{1}$ (or $\left.p_{1}\right)$ line. See for example eq. $(2,15)$ or $(2,16)$ and ref. $I$.

Clearly Fig, 7 represents a particular sequence of terms in the series (2. 9). While the notation of Figures 6 and 7 is rather compact, the reader should have no trouble generating this sequence. A typical term is shown in Fig. 8. Its contribution to eq. 2,9 has the form $T_{2}(E) b\left(E-H_{0}\right) \frac{\partial}{\partial E} T_{1}(E)$, where $T_{2}$ (and $T_{1}$ ) are unambiguously defined by the parts above (and below) the horizontal line in Fig. 8. The simple examples we did earlier in Fig. 4 and 5 also belong to the series in Fig. 7. 
It may be checked that every term contained in Fig. 7 involvek resonance propagators in only one independent energy variable, once full energy momentum conservation is imposed, just as in the examples (Figs, 4 and 5 ) we explicitly worked out. (We continue to retain only the imaginary part $-i \pi \delta\left(E-H_{0}\right)$ in cvery Green's function $\frac{1}{\mathrm{E}-\mathrm{H}_{0}+i \epsilon}$ in the $T$ matrices, It will be seen that these alter the width $\Gamma$. The real part of $\frac{1}{\mathrm{E}-\mathrm{H}_{0}+\mathrm{if}}$ will alter the resonance mass $M$ by an amount which vanishes as $g^{2} \rightarrow 0$ ) Thus, every term in the expansion of Fig. 7 will survive as $g^{2} \rightarrow 0$.

So far Fig. 7 has not included "exchanges" of spectator neutrons for pions) with the initial $P$ (or $k$ ) line. Let us now add, for every term in Fig. 7, also terms where either the neutron line $p$ or the pion line $k$ is exchanged with arbitrary numbers of spectators of the respective species. Thus, in the place of Fig. 7a, we use the whole sequence in Fig. 9. Similarly, 7b and $7 \mathrm{c}$ are expanded to include a trivially larger set when either $p$ or $k$ is "exchanged" an arbitrary number of times. Let us call the sum of all contributions in Fig. 7 along with such exchanges as $S$. S does not include terms, wheze both $p$ and k are exchanged with non-interacting lines. We will sum those separately and they will be seen to provide the "statistics" for the $\mathrm{N}^{*}$ Bpecies.

The set $\mathbf{S}$ has a simple sum, which has to be obtained by examining the structure of a general term such as Fig. 8 , and evaluating it as we did the simple case of $\mathrm{Fig.} \mathrm{4}$. It can be seen that the sum of all terms in $\mathrm{S}$ involving n resonances above the horizontal line, and $m$ resonances below the line $(\mathrm{n}=4, \mathrm{~m}=2$ in Fig. 8 ) is 


$$
\begin{aligned}
& -16 . \\
& S_{n, m}=\frac{\delta^{3}(0)}{2 \pi i} \sum_{p, k} e^{-\beta\left(E-\mu_{1}-\mu_{2}\right\rangle}\left\{1-\frac{e^{-\beta\left(e-\mu_{2}\right)}}{1+e^{-\beta\left(2-\mu_{2}\right)}}+\frac{e^{-\beta\left(\omega-\mu_{1}\right)}}{\left.1-e^{-\beta\left(\omega-\mu_{1}\right.}\right)}\right\}\left\{\begin{array}{c}
42^{2\left(-i \gamma_{N}+1 \gamma_{\pi}\right)^{n-1}} \\
\left(E-\sqrt{\left.M^{2}+p^{2}-i \Gamma\right)^{n}}\right.
\end{array}\right. \\
& \sum_{1} k_{1}\left\{1-\frac{e^{-\beta\left(e_{1}-\mu_{2}\right)}}{1+e^{-\beta\left(e_{1}-\mu_{2}\right)}}+\frac{e^{-\beta\left(\omega_{1}-\mu_{1}\right)}}{1-e^{-\beta\left(\omega_{1}-\mu_{1}\right)}}\right\} \delta\left(E_{1}-E\right) \delta^{3}\left(\vec{P}_{1}-\vec{P}\right) \\
& \frac{g}{\partial E}(2 \pi)^{4} g^{2} \frac{\left(+i \gamma_{N}-i \gamma_{\pi}\right)^{m-1}}{\left(E-\sqrt{\left.P^{2}+M^{2}+i \Gamma\right)^{m}}\right.}
\end{aligned}
$$

where $(p, k)$ and $\left(p_{1} k_{l}\right)$ are just the labels in Fig. 7 ,

$$
\begin{aligned}
& E=e+\omega, \bar{p}=\bar{p}+\bar{k}, E_{1}=e_{1}+\omega_{1}, \bar{P}_{1}=\bar{p}_{1}+\bar{k}_{1} \\
& Y_{N}=Y_{N}\left(E, P, \mu_{2}\right) \text { as in }(215) \\
& Y_{\pi}=Y_{\pi}\left(E, P, \mu_{1}\right) \text { as in }(Z, 16)
\end{aligned}
$$

Let us define a reduced wjdth

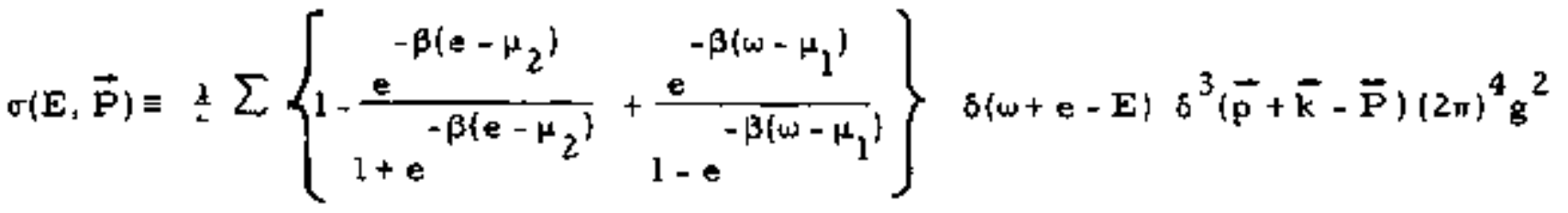

$$
\begin{aligned}
& =\left[\Gamma\{E, P\}-Y_{N}(E, P)+Y_{\pi}(E, P)\right]
\end{aligned}
$$

Thus,

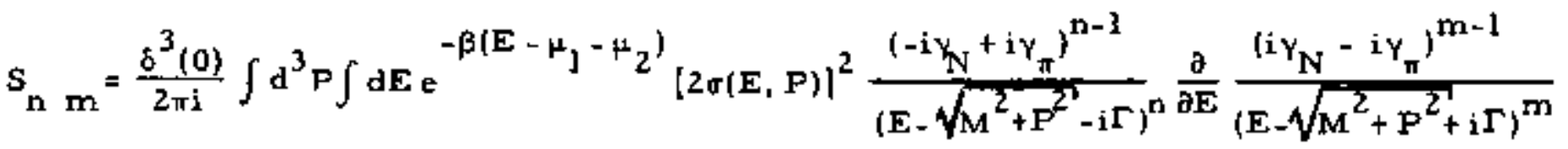

$\therefore S=\sum_{m, n=1}^{\infty} S_{m n}=\frac{\delta^{3}(0)}{2 \pi i} \int d^{3} P \int d E e^{-\beta\left(E-\mu_{1}-\psi_{2}\right)} \frac{2 \sigma(E, P)}{E-\sqrt{M^{2}+q^{2}}-i\left[\Gamma-Y_{N}+\gamma_{\pi}\right]}$

$$
\frac{-2 \sigma(E, P)}{\left[E-\sqrt{M^{2}+P^{2}}+i\left(\Gamma-Y_{N}+Y_{\pi}\right)\right]^{2}}
$$

Remembering that the only contribution, as $g^{2}-0$ comes at $E=\sqrt{M^{2}+P^{2}}$. 
$\Gamma$ in the denominators, which equals $\Gamma(M)=\Gamma\left(\sqrt{M^{2}+P^{2}}, P\right)$ can be replaced by $\Gamma(E, P)$. Then

$S=\frac{\delta^{3}(0)}{2 \pi i} \int d^{3} P \int d E e^{-\beta\left(E-\mu_{1}-\mu_{2}\right)} \frac{2 \sigma(E, P)}{\left[E-\sqrt{M^{2}+P^{2}}-i \sigma(E, P)\right]\left[E-\sqrt{\left.M^{2}+P^{2}+i \sigma(E, F)\right]^{2}}\right.}$

Note that (2.19) has the same simple form as the lowest virial coefficient evaluated in (2.10) corresponding to Fig. ib. except that the full width $\Gamma(E, P)$ has been replaced by a reduced width $\sigma(E, P)$. From its definition in (2. 18), we see that $\sigma(E, F)$ has a reduced phase space integral, where the occupation probability of the neutron has been subtracted, and that of the pion addedn. Such a reduction in width due to occupied fermions in the domain of decay and an enhancernent due to occupied bosonk, would be expected on physical grounds, when an $N^{*}$ decays in a medium. Our choice of diagrams contained in $S$ conspire to merely alter the width in a self consistent way. This is intuitively clear when a typical term in $S$, such as Fig. 8 is redrawn as in Fig. 10. Fig. 10 shows that all we are doing in the sequence $S$ is to expand the unitary series of the resonance in powers of the width $\Gamma$, and antisymmetrize the neutron and symmetrize the pion in the $\mathrm{N}^{*}$ wave function.

Finally, in the narrow $\left(\mathrm{g}^{2} \rightarrow 0\right)$ resonanco limit, where all the widths $\Gamma, Y_{N^{\prime}} Y_{\pi}$, and $\sigma$ go to zero anyway, this self consistent change in width should make no difference. This is clear from the fact that as $g^{2}$ (and hence $\left.\sigma(E, P)\right)-0$, using (2.8)

$S=\delta^{3}(0) \int d^{3} P \int d E e^{-\beta\left(E-\mu_{1}-\mu_{2}\right)} \delta\left(E-\sqrt{P^{2}+M^{2}}\right)=e^{+\beta\left(\mu_{1}+\mu_{2}\right)} v \int \frac{d^{3} P}{(2 \pi)^{3}} e^{-\beta\left(\mu^{2}+P^{2}\right)^{\frac{1}{2}}}$ $(2,20)$ 
Thus, $S$ merely reduces to the lowest virial term $e^{-\beta\left(\mu_{1}+\mu_{2}\right)} b(1,1)$. In the narrow resonance limit, all the other terms in $S$, which are individually nonzero, and belong to higher virial coefficients, simply cancel, leaving only the lowest contribution in $(2,10)$ due to the two particle $\pi^{-} \mathrm{n}$ scattering (Fig. lb). Let us now see what other contributions survive as $\mathrm{g}^{2}-0$, other than S. It can be checked that only two types of diagrams remain:

(1) Diagrams where both the $p$ and $k$ line in Fig. 6 are exchanged with. spectator particles. Remember that this was not included in S. Fig. 11 gives the sequence of such diagrams. Note that diagrams such as in Fig. 12 will also individually exist, but they add up to zero exactly for the same reason that higher virial terms in $\mathbf{S}$ cancelled. Thus the two examples in Fig. 12, apart from the extra exchanged pair which merely add a factor e $e^{-\beta\left(e_{1}^{+} \omega_{1}-\mu_{1}-\mu_{2}\right)}$ to both terms, are just two of the higher virial terms in $S$, all of which cancel to every order in $\mu_{1}$ and $\mu_{2^{*}}$ This leaves only terms of the type in Fig. 11. The reader can convince himself that diagrams in Fig. 11, along with the sequence $\$$ fof which only Fig. lb remains) exhaust all linked diagrams which survive as $g^{2}-0$. By "linked" we mean that all resonances in the diagram are manifestly connected by internal lines.

(2) In addition, one can also have unlinked terms which are connected because of the permutation of final state labels, and consequently are permitted in the partition function expansion. For appropriate permutation of labels, these will also have only one independent energy which resonates. See the examples. in Fig. 13. 
Note that the on-energy-shell $S$ matrix of two unconnected pieces is just a product of the individual $S$ matrices, each separately conserving energymomentum. It can be seen that, thanks to energy momentum conservation, all the resonances in Fign 13 occur in the same variable $\left\{e_{1}+\omega_{1}-\sqrt{M^{2}+\left(\vec{P}_{1}+\bar{x}_{1}\right)^{2}}+i I^{j}\right.$, so that such terms will survive upon integration.

We assert that diagrams exemplified in Fig. 13 and Fig, 11 , along with the sequence $S$, exhaust all possible linked and unlinked terms that effectively have only one independent resonant energy and therefore survive. To evaluate contributions in Fig. 11 and Fig. 13, let us consider for example $n_{1}=n_{2}=2$, when all terms of this type give

$$
\begin{aligned}
& \frac{1}{2 \frac{1}{2}} \frac{-1}{2 \pi i} \sum_{A B} e^{-\beta\left(e_{A}+e_{C}\right)+\beta\left(2 \mu_{1}+2 \mu_{2}\right)}<A\left|s_{1}^{-1}\right| B>C C\left|S_{2}^{-1}\right| D>\frac{\partial}{\partial\left(e_{A}+e_{C}\right)} \\
& \text { CD } \\
& \text { - }\left\{<\mathrm{B}\left|\mathrm{S}_{1}\right| \mathrm{C}><\mathrm{D}\left|\mathrm{S}_{2}\right| A>\right\}
\end{aligned}
$$

where labels A, B, C, D stand for pairs of $\pi^{-} n$ as in Fign 14, We have used the fact $t h e$ on energy shell $s$ matrices factorize for disconnected pieces - in this case into a product $S_{1} S_{2}$, We are using in $(2,21)$ on energy shell $S$ matrices as well as a derivative with respect to the total initial energy. Ref. 1 shows that this is equivalent to the off energy expression in (2.5) for unitary 5 matrices. Since $S_{1}$ and $S_{2}$ each conserve energy, it is clear for the particular set of exchanged labels in Fig. 14 that $e_{A}=e_{B}=e_{D}=e_{C}$. Further since each $S$ matrix is a sum of two terms $\langle i| s\} j\rangle=\delta_{j}-2 \pi i \delta\left(e_{i}-e_{j}\right) T_{i j}$, Fig. 14 is a sum of 16 terms, of which Fig. 13a and lia are examples. But the expression (2, 21) equals 


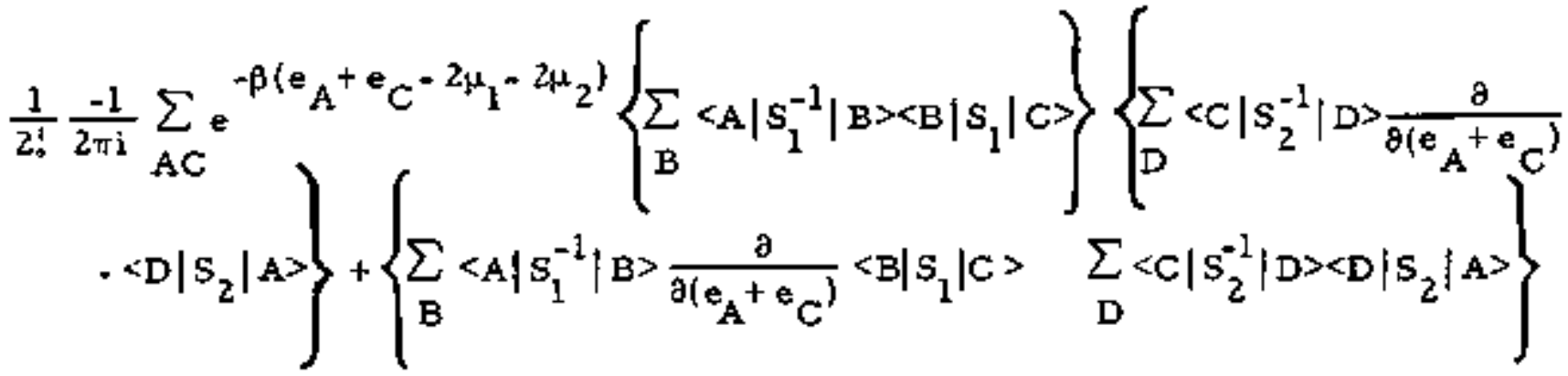

$$
\begin{aligned}
& =\frac{1}{2 !} \frac{-1}{2 \pi i} \sum_{A C} e^{-\beta\left(2 e_{A}-2 \mu_{1}-2 \mu_{2}\right)}\left\langle C\left|s_{2}^{-1} \frac{\theta}{2 \partial e_{A}} s_{2}\right| A\right\rangle \delta_{A C}+\delta \delta_{A C}\left\langle A\left|s_{1}^{-1} \frac{\partial}{2 \theta e_{C}} s_{1}\right| C\right\rangle
\end{aligned}
$$

where we have used $\frac{\partial}{\partial\left(e_{A}+e_{C}\right)}=\frac{\partial}{2 \partial e_{A}}=\frac{\partial}{2 \theta e_{C}}$

Thus, Fig, 14 merely gives

$\frac{1}{2 !} \cdot \frac{-1}{2 \pi i} \sum_{A} e^{-2 \beta\left(e_{A}-\mu_{1}-\mu_{2}\right)}<A\left|S^{-1} \frac{\partial}{\partial E} s\right| A>=\frac{-1}{2 !} v \int \frac{d^{3} P}{(2 \pi)^{3}} e^{-2 \beta\left(\sqrt{P^{2}+M^{2}}-\mu_{1}-\mu_{2}\right)}$

following earlier algebra.

Incidentally, the $\frac{1}{2 !}$ in (2. 2l) is the usual Boltzmann factor since there are two equivalent pairs in Fig. 14, while the minus sign in front ia becalae a pair of fermions and a pair of bosons have been exchanged in the final state.

Following the same method, it can be seen that unlinked terms with $n_{1}=m$ pions and $n_{2}=m$ neutrons will yield

$$
\frac{(-1)^{m-1}}{m} \vee \int \frac{d^{3} P}{(2 \pi)^{3}} e^{-\beta m\left(\sqrt{P^{2}+M^{2}}-\mu_{1}-\mu_{2}\right)}
$$

The replacement of $\frac{1}{m !}$ by $\frac{1}{m}$ is because there are (m-1)! permutations-of the final $\mathrm{nm}^{-}$pairs that can keep m links "connected," Summing 2, 23 over m, the number of $\pi^{-} n$ pairs, we have for the full expansion $(2,9)$

$$
\log Z=\log Z_{0}+V \int \frac{d^{3} P}{(2 \pi)^{3}} \log \left[e^{-\beta\left(\sqrt{P^{2}+M^{2}}-\mu_{1}-\mu_{2}\right)}+1\right]
$$


All contributions to all the virial coefficients in (2.9) have been included in (2. 24). The entire sequence of linked terms in what we called $S$, merely readjusted the resonance width in a self consistent way, as shown in eq. (2, 19). giving us back just the contribution of the lowest virial term (2, 10). This. forms the $m=1$ case of the expression 2,23 , Unlinked terms with $m$ pieces gave (2, 23) for the appropriate value of $\mathrm{m}$, and the sum over $\mathrm{m}$ yield (2. 24). There are no other contributions to any of the virial terms as $g^{2}-0$.

The result $(2,24)$ is exactly what one would desire intuitively, vizn, that the interaction between the $\pi^{-}$n system, in our model, is replacable by a third species of $\mathrm{N}^{*}$ particles, with mass $M$, with Fermi statistics, and a chemical potential $\mu_{\mathrm{N}^{*}}=\mu_{1}+\mu_{2}$, as required by Baryon and charge conservation. Apart from this constraint on the chemical potential (which will determine the relative "density" of $\mathrm{N}^{*}$ to those of $\mathrm{n}$ and $\pi^{-}$for a given temperature, charge and Baryon number), the $\mathrm{N}^{*}$ species is independent of the $\mathrm{n}$ and the $\pi$. It's states need not be antisymmetrized in any sense with those of the neutrons, or symmetrical with those of the pions. All this is true only in our model of a purely narrow resonance dominated dynamics - an assumption we examine in the following paper. 


\section{A FAMILY OF RESONANCES}

We now extend these ideas to more general situations. When narrow resonances scatter with other particles or other narrow resonances, we show that the resulting contributions to the partition function $Z$ behave as if the resonances were elementary stable particles. Secondly, when resonances scatter with other particles and resonances, only to produce yet other narrow resonances, i. e., when all n particle scattering happens through "tree graphs" as in Fig. 15, then

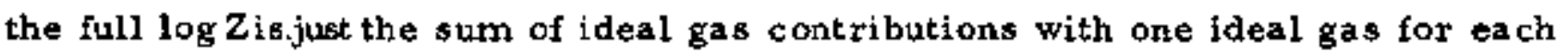
separate type of resonance. Any specific process, such as Fig. 15, contributes an ideal gas term corresponding to the super $n$-particle resonance from which all the initial and final particles cascade.

We prove these assertions for the simple example of 3 -particle scattering in a Boltzmann gas. Let particles 1 and 2 interact only through a narrow resonance $N_{12}^{*}$, which in turn can scatter of ${ }^{\prime}$ particle 3 (Fig. 15). More precisely, let the three particle $T$ matrix be given by

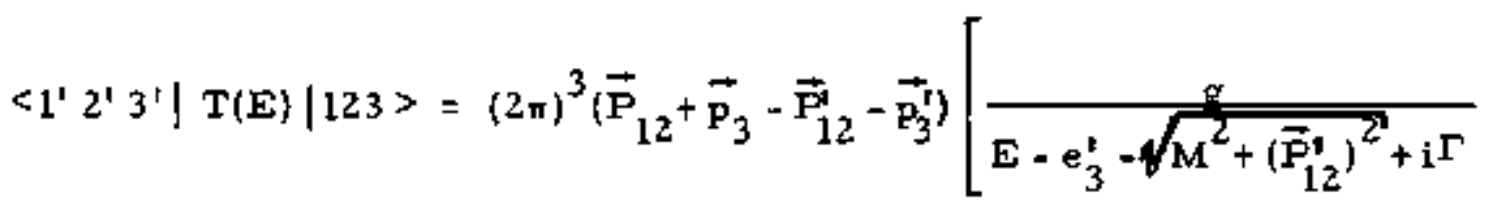

$$
\begin{aligned}
& \text { - }\left\langle\mathrm{P}_{12}^{i} \mathrm{p}_{3}^{\prime}|\tau(\mathrm{E})| \mathrm{P}_{12}, \mathrm{P}_{1}>\frac{\mathrm{g}}{\mathrm{E}-\mathrm{e}_{3}-\sqrt{\left(\overrightarrow{\mathrm{P}}_{12}\right)^{2}+\mathrm{M}^{2}+\mathrm{i} \Gamma}}+\frac{\mathrm{g}^{2} \delta^{3}\left(\overrightarrow{\mathrm{p}}_{3}-\overrightarrow{\mathrm{p}}_{3}^{1}\right)}{\mathrm{E}-\mathrm{e}_{3}-\sqrt{\mathrm{M}^{2}+\left(\overrightarrow{\mathrm{P}}_{12}\right)^{2}+i \Gamma}}\right]
\end{aligned}
$$

Here, the resonance parameters $M, g^{2}$ and $\Gamma$ are as defined earlier (2.3), $P_{12}=\left(\vec{P}_{12}, E_{12}\right)=p_{1}+p_{2}$, and $P_{12}^{\prime}=\left(\vec{P}_{12}^{\prime}, E_{12}^{\prime}\right)=P_{1}^{t}+p_{2}^{\prime}$. The second term in (3.1) corresponds to Fig. 16b, where particle 3 does not interact at all. This clearly has also to be included in $T(E)$ if Fig. $16 a$ is included. Before inserting 
(3.1) inlu the virial series, let us examune untarity for $T(E)$. As $g \rightarrow 0$, $T(E)$ vanishes, as per the identity (2.7). So does $\mathrm{T}^{+}(\mathrm{E})$. Therefore unitarity reduces to

$$
T^{+}(E) \delta\left(E-H_{B}\right) T(E)=0
$$

Now take for example (3.2) a applied to forward scattering $T^{+}(E) \delta\left(E-H_{0}\right) T(E)$ has four contributions (Fig, 17) where once again Fig. 17d vanishes as g $\rightarrow 0$. It is just the imaginary part of Fig. 16b. But the remaining terms (Figs, 17a, b,c) do not vanish as $\mathrm{g} \rightarrow 0$. Followng the simple algebra of narrow resonances as in the last section, it is easy to check that (3.2) merely yields, when (3.1) is substituted for $T(E)$ and the limit $g^{2} \rightarrow 0$ taken,

$0=$ Fig. $(17 a+17 b+17 c)$

$$
\begin{aligned}
& =(2 \pi)^{4} \delta^{3}(0) \delta\left(E_{12}-\sqrt{P_{12}^{2}+M^{2}}\right)\left(\frac{(2 \pi)^{4} E^{2}}{2 \Gamma}\right) \\
& \text { - }\left[\left\langle\mathrm{P}_{12} \cdot \mathrm{P}_{3} \int_{\mathrm{i \tau}}{ }^{\dagger}-\mathrm{i} \tau \mid \mathrm{P}_{12}, \mathrm{P}_{3}\right\rangle+2 \pi \int \mathrm{d}^{3} \mathrm{P}_{3}^{\prime} \mathrm{d}^{3} \mathrm{P}_{12}^{\prime} 5^{3}\left(\mathrm{P}_{12}+\mathrm{P}_{3}-\mathrm{P}_{12}^{\prime}-\mathrm{P}_{3}^{\prime}\right)\right. \\
& \left.\left.\left.\delta^{0}\left(\sqrt{P_{12}^{2}+M^{2}}+e_{3}-\sqrt{\left(P_{12}^{\prime}\right)^{2}+M^{2}}-e_{3}^{\prime}\right)<P_{12} p_{3}\left|\tau^{+}\right| P_{12}^{\prime} p_{3}^{\prime}><P_{12}^{\prime} p_{3}^{\prime}\right] \tau\right] P_{12} p_{3}>\right]
\end{aligned}
$$

It is not surprising that the relation $\mathrm{T}^{+} \mathrm{T}=0$, when expanded yields the familiar two particle unitarity relation for $\tau(E)$, on the resonance mass shell $E_{12}^{2}=P_{12}^{2}+M^{2}$

Now, let us go on to evaluate the virial contribution to log $Z$ due to the $T$ matrix in $(3,1)$. As in the last section, the $\frac{\partial}{\partial \mathrm{E}} \mathrm{T}(\mathrm{E})$ term will vanish due to identity (2.7) leaving only the $T^{+}(E) \frac{\partial}{\partial E} T(E)$ term as in $(2,9)$. For our 
scattering amplitude (3.1), this gives three contributions, which can again be represented by the same Figures $17 \mathrm{a}, 17 \mathrm{~b}$, and $17 \mathrm{c}$, with the understanding that $\frac{\partial}{\partial \mathrm{E}}$ acts on the lower part of each diagram. [Note that Fig. 17d being disconnected is not to be included in the virial series. The ideal gas term due to the resonance $\mathrm{N}_{12}^{*}$ is already present in the lower (two-particle) virial coefficient in $(2.10)$,

The amplitude $T(E)$ in $(3,1)$ depends on $E$ through $\tau(E)$ as well as the resonance propagators. When $\frac{\partial}{\partial E}$ operates on $\left\{6\left(E-H_{0}\right) T(E)\right\}$ as required in the virial expansion, it will give terms where the $\mathrm{N}_{12}^{\text {t* }}$ resonance propagator is differentiated, and other terms where the resonance propagator is not differentiated. Let us begin with the former set of ferms. This contribution from Figures $17 a, b$, and c to the virial series can be seen to be proportional to

$\sum_{123} \frac{\delta\left(E_{12}^{2}-\sqrt{\left.\left(\vec{P}_{12}\right)^{2}+M^{2}\right)}\right.}{i \Gamma}\left[i<P_{12} p_{3}\left|\tau^{+}-\tau\right| P_{12} p_{3}>+2 \pi \int d^{3} P_{12}^{t} \int d^{3} p_{3}^{\prime} \delta^{3}\left(\vec{P}_{12}+\vec{p}_{3}-\bar{P}_{12}^{\prime}-\bar{p}_{3}^{\prime}\right)\right.$ - $\left.\left.5\left(\sqrt{\overrightarrow{\mathrm{P}}_{12}^{2}+\mathrm{M}^{2}}+\mathrm{e}_{3} \cdot \sqrt{\left(\overrightarrow{\mathrm{P}}_{12}^{\prime}\right)^{2}+\mathrm{M}^{2}}-\mathrm{e}_{3}^{\prime}\right)<\mathrm{P}_{12} \mathrm{P}_{3}\left|\tau^{+}\right| \mathrm{P}_{12}^{\mathrm{t}} \mathrm{P}_{3}^{\prime}\right\rangle\left\langle\mathrm{P}_{12}^{\prime} \mathrm{P}_{3}^{\prime}|\tau| \mathrm{P}_{12} \mathrm{P}_{3}\right\rangle\right] \ldots$

This is essentially the unitarity constraint in (3.3) except for the extra factor $\frac{l}{i \Gamma}$ which occurs because the $\frac{\partial}{\partial E}$ factor operates on the resonance propagator. From (3.3) then, the contribution (3.4) vanishes by unitarity, even though any single term in it would diverge as $r-0$.

We are left with contributions where $\frac{\partial}{\partial \mathrm{E}}$ differentiates the product $\delta\left(E-H_{0}\right) \tau(E)$, leaving the resonance propagators alone. These clearly exist only for Fig. 17b and $17 c$. These give for the virial series $(2,5)$, 
Fig. $17 b=\frac{\delta^{3}(0)}{2 \pi i} \int d E \sum_{123} e^{-\beta\left(E-\sum_{i} \mu_{i}\right)} \frac{(2 \pi)^{4} g^{2}}{(x-i \Gamma)(x+i \Gamma)} \sum_{1^{\prime} 2^{1}} \cdot \delta^{4}\left(P_{12}^{1}-P_{12}\right) \frac{(2 \pi)^{4} g^{2}}{y+i \Gamma}$

$$
\text { - } \left.\frac{\partial}{\partial E}\left\{\delta\left(E-E_{12}-e_{3}\right)<P_{12}^{t} p_{3} \mid \tau(E)\right] P_{12} p_{3}>\right\}
$$

where $x=E_{12}-\sqrt{\vec{P}_{12}^{2}+M^{2}}, \quad y=E_{12}^{1}-\sqrt{\left(\vec{P}_{12}^{\prime}\right)^{2}+M^{2}}$ and $\mu_{i}$ are the three chemiral potentials, As $g^{2}-0$, this gives

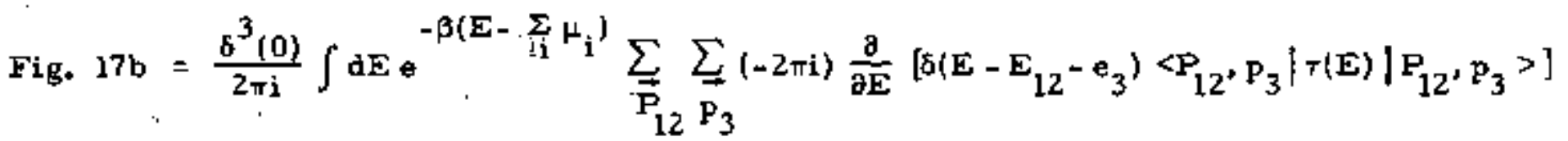

where $\mathbf{E}_{12}^{2}$ is constrained to be $\cdot\left(\overline{\mathrm{P}}_{12}^{2}+\mathrm{M}^{2}\right)$

Similarly, Fig. $17 \mathrm{c}$ will yield, when $\frac{\partial}{\partial E}$ acts on $\delta\left(E-H_{0}\right) T(E)$

Fig. $17 \mathrm{c}=\frac{\delta^{3}(0)}{2 \pi \mathrm{i}} \int \mathrm{dE} \mathrm{e}^{-\beta\left(E-\sum_{\mathrm{i}} \mu_{\mathrm{i}}\right)} \sum_{\mathrm{P}_{12} \mathrm{P}_{3}} \sum_{2 \pi \delta\left(E-E_{12}-e_{3}\right)} \sum_{\mathrm{P}_{12}^{\mathrm{l}} \mathrm{p}_{3}^{\mathrm{i}}} \sum_{12^{2}}\left\langle\mathrm{P}_{3} \mathrm{p}^{+}(\mathrm{E}) \mid \mathrm{P}_{12}^{1} \mathrm{P}_{3}^{1}\right\rangle$

$$
\frac{\partial}{\partial E}\left[2 \pi \delta\left(E_{12}+E_{3}-E_{12}^{\prime}-e_{3}^{\prime}\right] \delta^{3}\left(P_{12}+P_{3}-P_{12}^{\prime}-p_{3}^{\prime}\right)\left\langle P_{12}^{\prime} P_{3}^{\prime}|\tau(E)| P_{12} P_{3}^{\prime}\right\rangle\right]
$$

where again $\left(E_{12}^{\prime}\right)^{2}=\left(\vec{P}_{12}^{t}\right)^{2}+M^{2}, E_{12}^{2}=\left(\vec{P}_{12}\right)^{2}+M^{2}$

In deriving (3.6), the resonance propagators have been combined . to form $\delta$ functions in the variables $y$ and $x$, which restrict the pairs 12 and $1^{\prime 2}$ ' to a total mass M. The results (3.5) and (3.6) are just what ithe scatter ing of particle 3 with a stable particle of mass $M$, momentum $\bar{r}_{12}$ and chemical potential $\mathrm{H}_{1}+\mathrm{H}_{2}$ would yield. Now take the special case when the $\mathrm{N}_{12}^{*}$-particle 3 scattering is itself dominated by a narrow resonance $N_{123}^{*}$ (Fig. 18). That is, let

$$
\left\langle\mathrm { P } _ { 1 2 } \mathrm { P } _ { 3 } \left(\tau\langle E)\left|\mathrm{P}_{12}^{1} \mathrm{P}_{3}^{1}\right\rangle=\frac{(2 \pi)^{3} \mathrm{G}^{2}}{\mathrm{E}-\sqrt{\mathcal{J}^{2}+\left(\overrightarrow{\mathrm{P}}_{12}+\overrightarrow{\mathrm{P}}_{3}\right)^{2}}+\mathrm{iY}}\right.\right.
$$


with $2 y=\sum_{\vec{P}_{12}} \overrightarrow{\vec{P}}_{3} \delta^{3}\left(\overrightarrow{\mathrm{P}}_{12}+\overrightarrow{\mathrm{p}}_{3}\right) \delta\left(\overrightarrow{\mathrm{P}}_{12}^{2}+\mathrm{M}^{2}+\mathrm{e}_{3}-3 \mathrm{~h}\right)(2 \pi)^{4} \mathrm{G}^{2}$. Then, as $\mathrm{G}^{2} \rightarrow 0$, the virial contributions in (3.5) and (3.6) follow the same pattern as the lowest virial term in Fig, 1 and eq. $\{2,10)$. The contribution in (3,5) vanishes due to identity (2,7) and in (3,6) only the term where the derivative $\frac{\partial}{\partial E}$ acts on $T(E)$ survives. This aurviving contribution to $(3,6)$ is just $\frac{\delta^{3}(0)}{2 \pi i} \sum_{\vec{p}_{12}, \overrightarrow{\mathrm{p}}_{3}} e^{-\beta\left(\sqrt{\left(\overrightarrow{\mathrm{P}}_{12}\right)^{2}+\mathrm{M}^{2}}+e_{3}-\Sigma_{\mu_{i}}\right)} \sum_{\overrightarrow{\mathrm{P}}_{12}^{\prime}, \overrightarrow{\mathrm{P}}_{3}^{\prime}} \delta^{4}\left(\mathrm{P}_{12}+\mathrm{P}_{3}-\mathrm{P}_{3}^{\prime}-\mathrm{P}_{12}^{\prime}\right) \frac{\left[(2 \pi)^{4} \mathrm{G}^{2}\right]^{2}(-1)}{(Z+i Y)(Z-i Y)^{2}}$

where $z \equiv \sqrt{\vec{P}_{12}^{2}+M^{2}}+e_{3}-\sqrt{M^{2}+\left(\vec{P}_{12}+\vec{p}_{3}\right)^{2}}$

Integrating $(3.8)$ over all variables except $\overrightarrow{\mathrm{P}}_{123}=\overrightarrow{\mathrm{P}}_{12}+\overrightarrow{\mathrm{p}}_{3}$ and $\mathrm{E}_{123}=$ $\sqrt{\left(\overrightarrow{\mathrm{P}}_{12}\right)^{2}+\mathrm{M}^{2}}+\mathrm{e}_{3}$, we obtain when $\mathrm{G}^{2}$ and $\mathrm{Y}$ tend to zero, the result $\delta^{3}(0) \int d^{3} \overline{\mathrm{P}}_{123} \int d E_{123} e^{-\beta\left(E_{123}-\sum_{i} \mu_{i}\right)} \delta(2)$
$=V \int \frac{d^{3} \bar{P}_{123}}{\{2 \pi)^{3}} e^{-\beta \sqrt{\left(\bar{P}_{123}\right)^{2}+M^{2}}+\Sigma \beta \mu_{i}}$

Thus, the scattering through the "super" resonance $\mathrm{N}_{123}^{*}$ again produces an "ideal gas" term corresponding to mass $\mathrm{J} / \mathrm{h}$ and chemical potential $\mu_{1}+\mu_{2}+\mu_{3}$ ' in addition to the ideal gas term corresponding to the two particle resonance $\mathrm{N}_{12}^{*}$ present in the lowest virial coefficient.

These results have been derived only for three-particle scattering of a Boltzmann gas. This was jugt to keep the algebra simple. It is clear from the proof that the generalization of eq. (3.5) and (3.6) where a narrow resonance acatters just like a stable particle, and of $(3,9)$ where a super resonance formed out of lesser resonances again gives an ideal gas term to more than 
three Boltzmann particles will be straightforward. One must merely keep track of all eonnected and disconnected pieces of the $S$ matrix, and the width of any given resonance will have to include the phase space of all the channels connected to it. Generalization to Bose or Fermi systems will of course be complicated. In Section $\mathrm{IL}$, where scattering took place only by forming one type of two-particle resonance, we saw that certain exchange diagrams exist in all virial coefficients. The only "direct" diagram in that case was Fig. lb. which would have been the gole contributor had the particles obeyed Boltzmann statistics. In the Boson-Fermion case dealt with in Section II, the exchange diagrams required a considerable: amount of diagrammatic analysis and narrow resonance algebra, But the net result was simple and physically reasonable. The higher exchange terms merely altered the width self consis. tency, to account for the fact that the resonance decays in a medium, and provided the resonance "particle" with approximate statistics. The many. particle dynamics of Section $\amalg$, when applied to Bose and Fermi systems will again yield a family of exchange diagrams in higher virial coefficients. But we hope the reader will be convine that their effect will once again be to merely provide the appropriate statistics to all the resonances. , 


\section{CONCLUSION}

We showed in Section II that a narrow elastic tesonance in a $\pi^{-} n$ channel behaves like an elementary particle with Fermi statistics. It is clear that this result is more general. If the initial stable particles were both bosons or both fermions, the corresponding changes in sign of exchange diagrams will ensure that the resonance is a boson. Inelastic regonances will again give the same result. Suppose a resonance had two decay channels. (This cannot happen in the examples of Section II where " $n$ " and "T " had separately conserved quantum numbers, but it can if $\pi^{+}$were included:) Let the $\mathrm{N}^{*}$ decay into

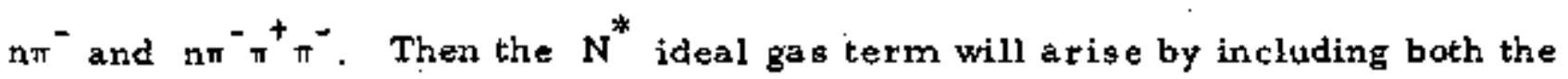
$\mathrm{n} \pi^{-}$and the $n \pi^{-} \pi^{+} \pi^{-}$states in the initial and intermediate states of Fig. 1 . Both these states have the same chemical potential, and are both to be included in the width of the $N^{*}$. Thus, our result that narrow resonances act like a separate species generalizes to all choices of quantum numbers, for elastic and inelastic resonances.

In both the previous sections, we always started with idealized manyparticle scattering amplitudes - those involving only narrow resonances. Thus the elastic two-particle amplitude in Section $I$ had only a pole at the resonance energy and nothing else. This is not to imply that any real system has such simple dynamics. In fact, as we discuss in the following paper, such a simple amplitude is forbidden in pocential theory.

To the extent that a real system has wide resonances, non-resonant scattering in addition, or no resonances at all, its thermodynamics will have to be calculated the usual hard way. This is abvious and not germane to our interest here. Instead, we wanted to verify the notion that to the extent that 
scattering in some case of interest is dominated by narrow resonances, it could be replaced by additional ideal gas terms with the quantum numbers of the resonances. This is one concrete way of stating the vague equivalence of narrow resonances with stable particles. We found that in fact such a replacement can be made in precisely the way expected intuitively.

When a resonance is wide, as compared to $\mathrm{k} T$, (this is sofor the $\triangle$ resonance in neutron stars\} then two types of corrections need to be made. A trivial correction is that expressions such as $\frac{\Gamma}{(E-i \Gamma)(E+i \Gamma)}$ cannot be replaced by $\pi \delta(E)$, and will have to be integrated more accurately, More geriously, the vast families of diagrams and contributions we discarded will now contribute. An example is a process like Fig. 3, which can be considered as repeated resonance particle scattering. For a wide resonance large coupling $\mathrm{g}^{2}$ ), such scattering will become important, and will have to be corrected for. This is of course very difficult in practice, but estimates a re being made in neutron star calculationa involving the 3-3 resonance $\Delta$, to correct for $\Delta=n$ and $\triangle-\triangle$ scattering. 5

We are grateful to Prof. S. B. Treiman for a useful discussion. 
$-30-$

REFERENCES

1. R. Dasher, S. Ma, and H. J. Bernstein, Phys, Rev. 187, 345 (1969);

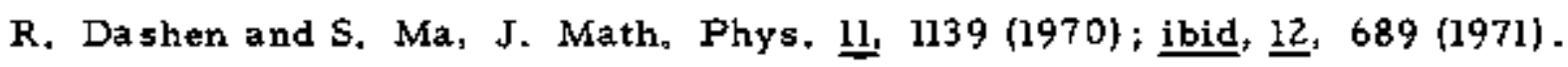

2. K, Huang. Statistical Mechanic g, John Wiley and Sons, Inc. New York (1963).

3. P. D. B. Collins, Phys. Reports 1C, 103 (1971).

4. R, Hagedorn, Suppl. Nova Cimento $\underline{3}, 147(1965)$; Aston. and Astrophys. $\underline{5}$, $184(1970)$.

5. H, A. Bethe and M, Johnson, (Private Communication). 


\section{FIGURE CAPTIONS}

Fig. 1 (a) Narrow resonance $N^{*}$ in the $\pi^{-} n$ channel and (b) its contribution to the lowest virial coefficient. Fig. lb stands for a $T^{+} \frac{\partial}{\partial E} T$ form as occuring in eq. 2.9 , where the horizontal line separates $\mathrm{T}^{+}$from $\mathrm{T}$ and an operation $\frac{\partial}{\partial \mathrm{E}}$ is to be applied on the piece $T$ below the horizontal line. In this and subsequenl diagrams, dashed lines, regular lines and double lines stand for pions, neutrons and the resonance $\mathbb{N}^{*}$ respectively.

Fig. 2 (a) A typical many particle $S$ matrix element in resonance dominated dynamics. This is a term in a two pion - three neatron scattering. (b) A typical T matrix diagram not permitted since two successive resonances occur between the same $\pi^{-} n$ pair. A unitary resonance as in (2. 1) already includes such iterations.

Fig. 3 A contribution to the one pion - two neutron $T$ matrix, which stands for the expression in eq. 2.4.

Fig. 4 Examples of diagrams which have only independent resonance energy, consequently surviving in the virial series. Such diagrams will end up refinring the $\mathrm{N}^{*}$ width in the presence of other neutrons in the medium,

Fig. 5 Corresponding examples to Fig. 4, but where the pions are "exchanged." These will help increage the $N^{*}$ width due to the presence of other pions in the medium. 
Fig. 6 An iterative sequence which generates T matrix elementa with only one independent resonance energy. This exhausts all linked $T$ matrices having this property. We use the phrase "'linked" to include trivially disconnected pieces consisting of non-interacting lines which are exchanged.

Fig. 7 An iterative sequence for $T^{+} \frac{\partial}{\partial E} \mathrm{~T}$ obtained by substituting the sequence in Fig. 6 for the shaded blob.

Fig. 8 An example of a typical term contained in Fig. 7. This particular example is contained in Fig. 7a,

Fig. 9 This is essentially the sequence in Fig. $7 a$, where, in addition, either the initial neutron, or pion (but not both) is exchanged with an arbitrary number of non-interacting spectatora, A similar enlargement on Fig. $7 \mathrm{~b}$ and Fig. $7 \mathrm{c}$ is also to be made. The net sum is called $S$.

Fig. 10 This is Fig. 8 redrawn to bring out the reas on why such diagrams merely alter the width.

Fig. 11 The sequence where both the initial pion and neutron are exchanged with spectators, starting from the primative diagram in Fig. lb.

Fig. 12 The corresponding sequence for higher virial terms of S. These however cancel for the same reason that the higher virial terma in $S$ cancelled. See the text. 
Fig. 13 Examples of unlinked contributions, which are however connected by the exchange of labels. Note that for the permutation of labels that connects such diagrams, all resonances again occur in the same energy variable. Thus every such contribution survives.

Fig. 14 The total contribution from two tanlinked pieces.

Fig. 15 Scattering of many particles through "tree graphs" made of only narrow resonances and stable particles.

Fig. 16 The 3-particle $T$ matrix where particles 1 and 2 can form a narrow resonance, which may (Fig. a) or may not (Fig. b) interact with 3.

Fig. 17 The four graphs representing $T^{+} T$ corresponding to the two terms in Fig. 16 for $T(E)$. These same graphs will be used to represent $\mathbf{T}^{+} \frac{\partial}{\partial \mathbf{E}} \mathrm{T}$ as well.

Fig. 18 The scattering of the resonance $\mathrm{N}_{12}^{*}$ with particle 3 through another narrow resonance $\mathrm{N}_{123}^{*}$. 

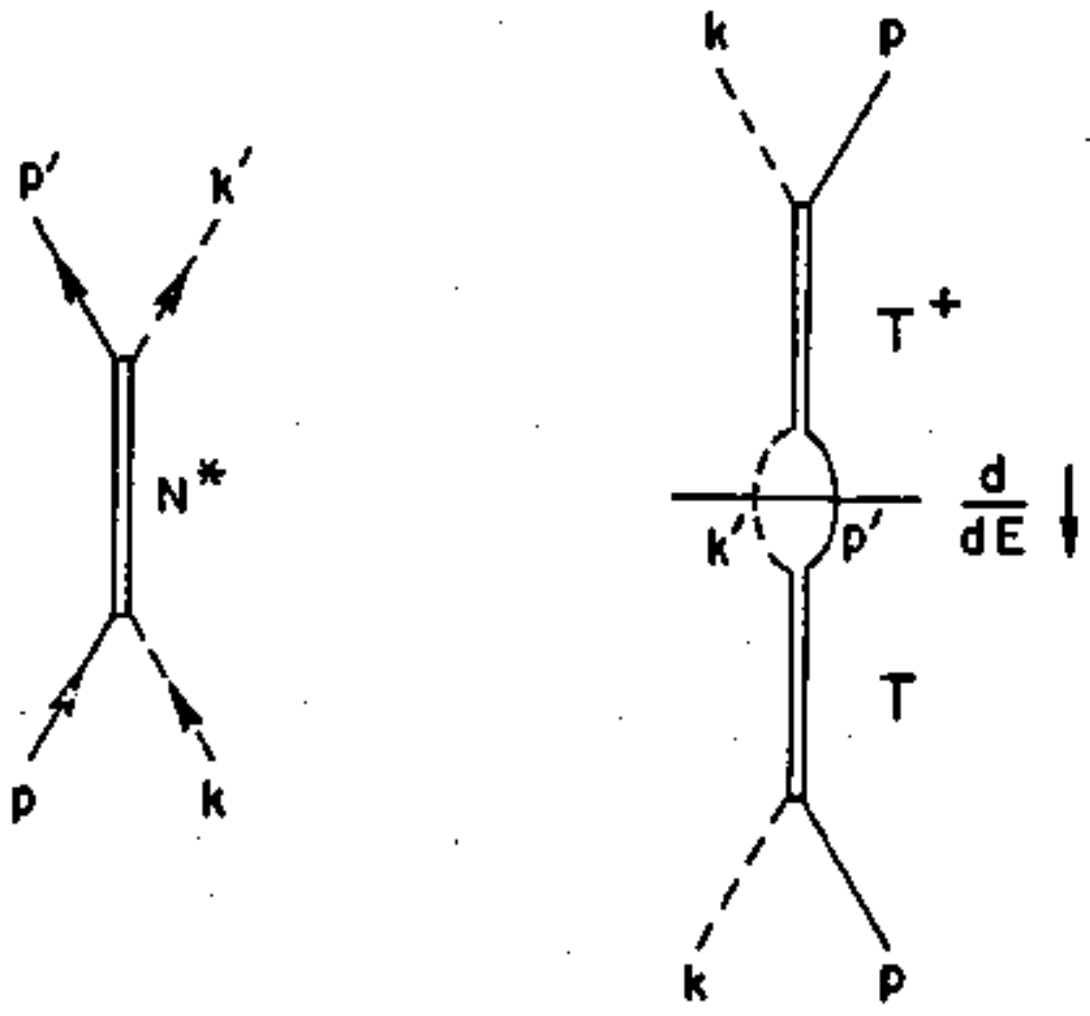

(a)

(b)

FIGURE 1 


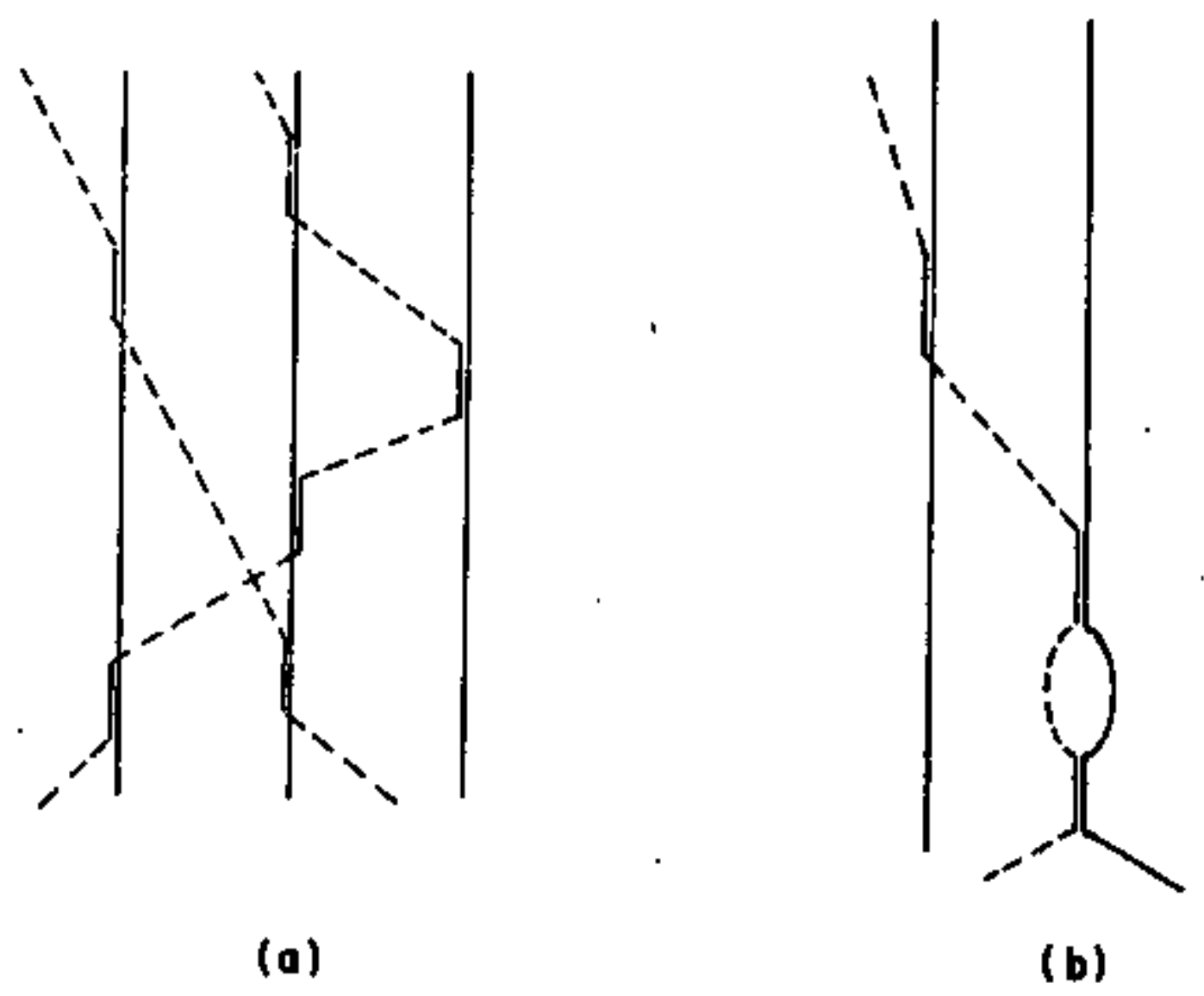

FIGURE 2 


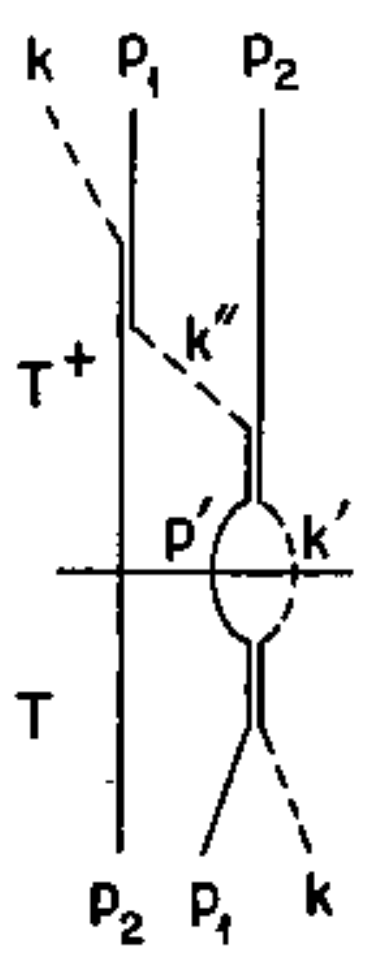

(a)

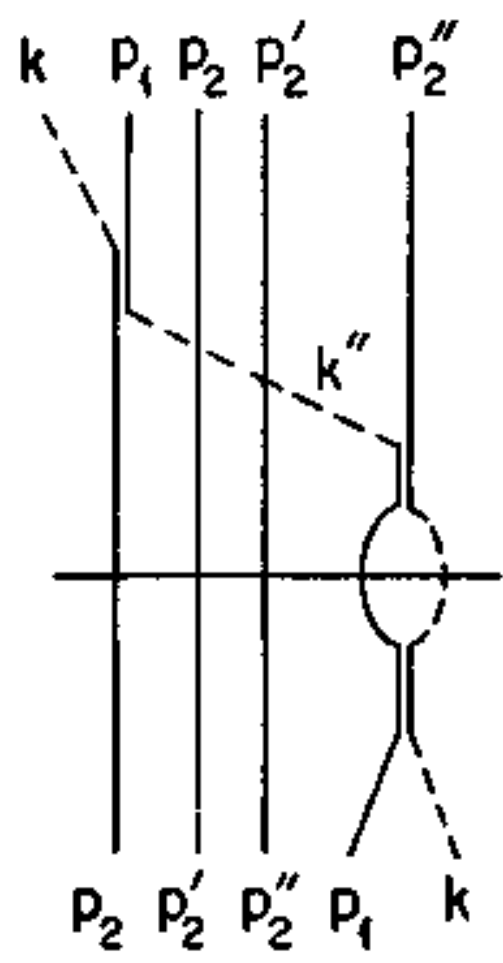

(b)

FIGURE 4 



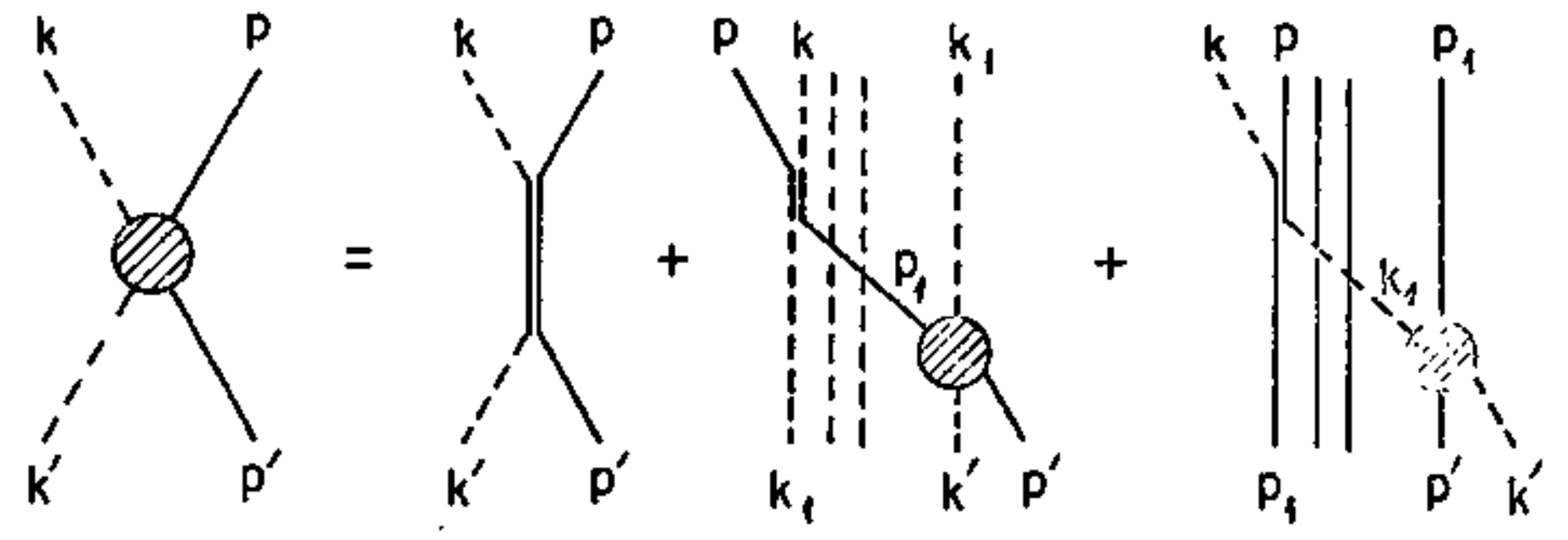

FIGURE 6 


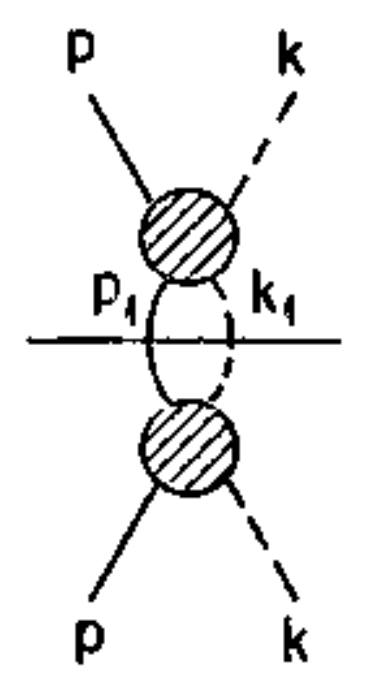

(a)

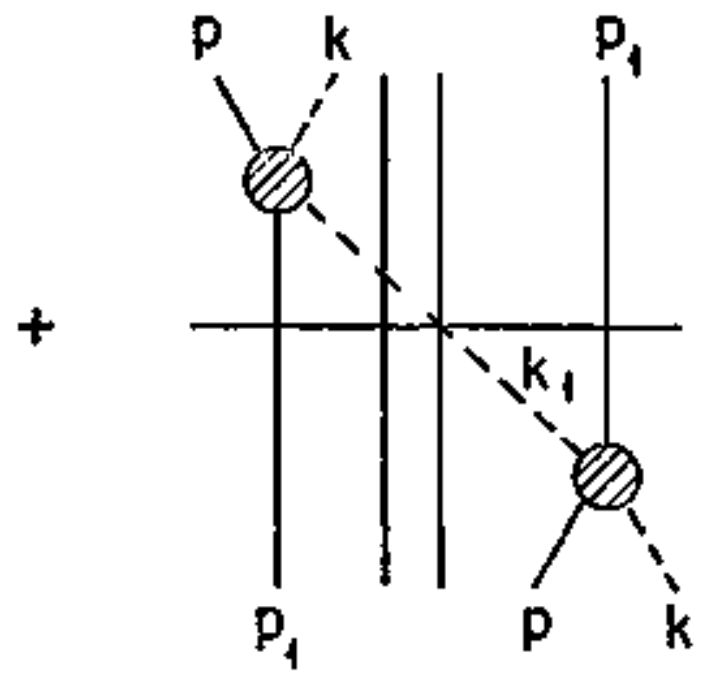

(b)

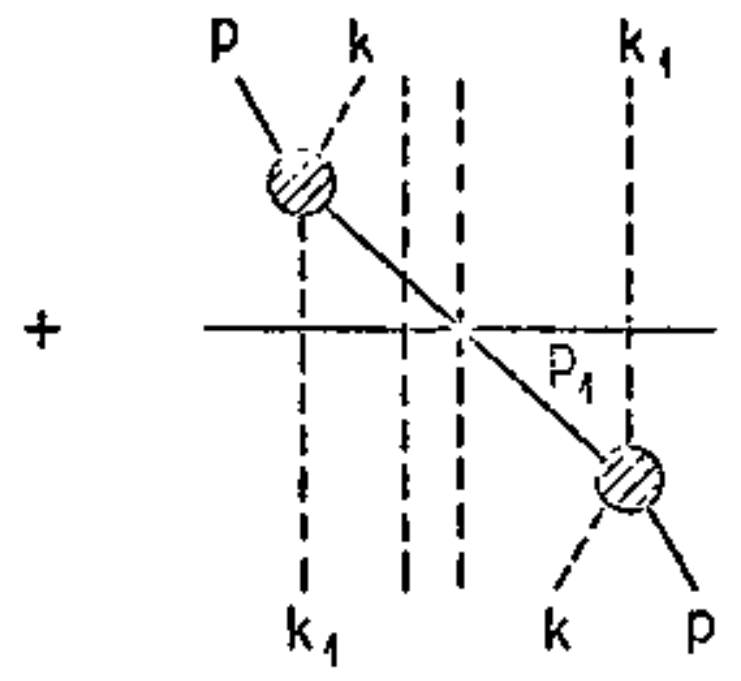

(c)

FIGURE 7 


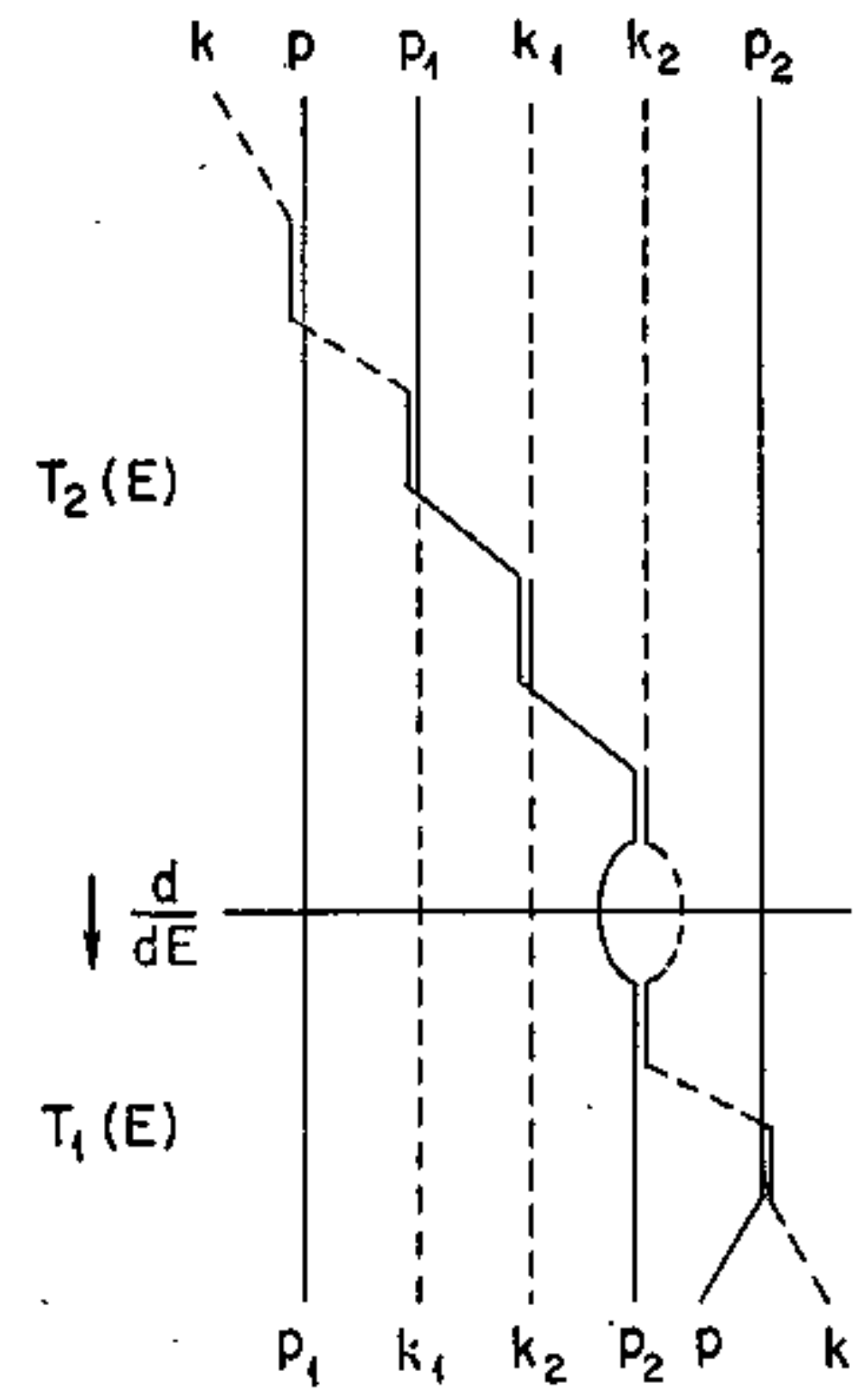

FIGURE 8 

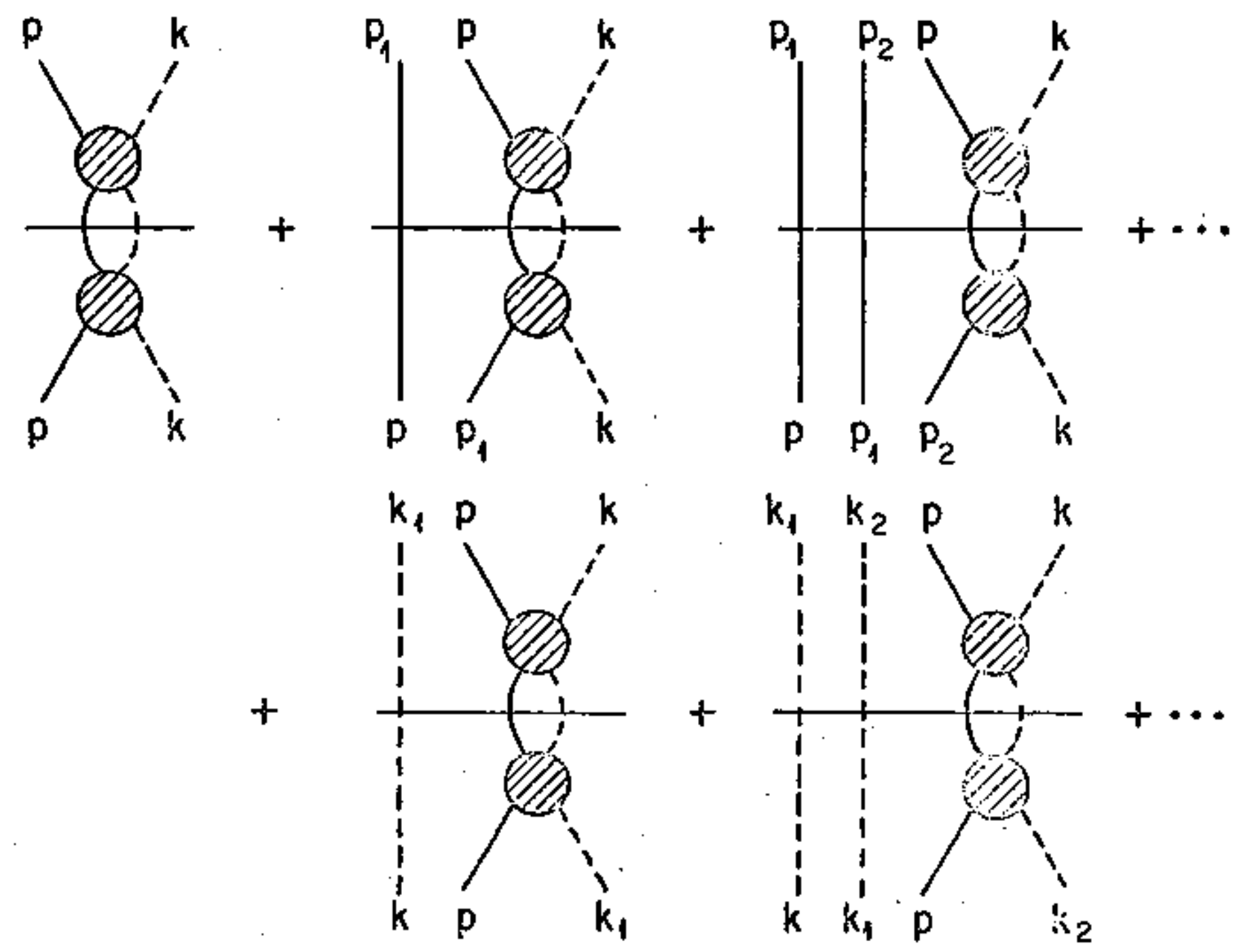

FIGURE 
I

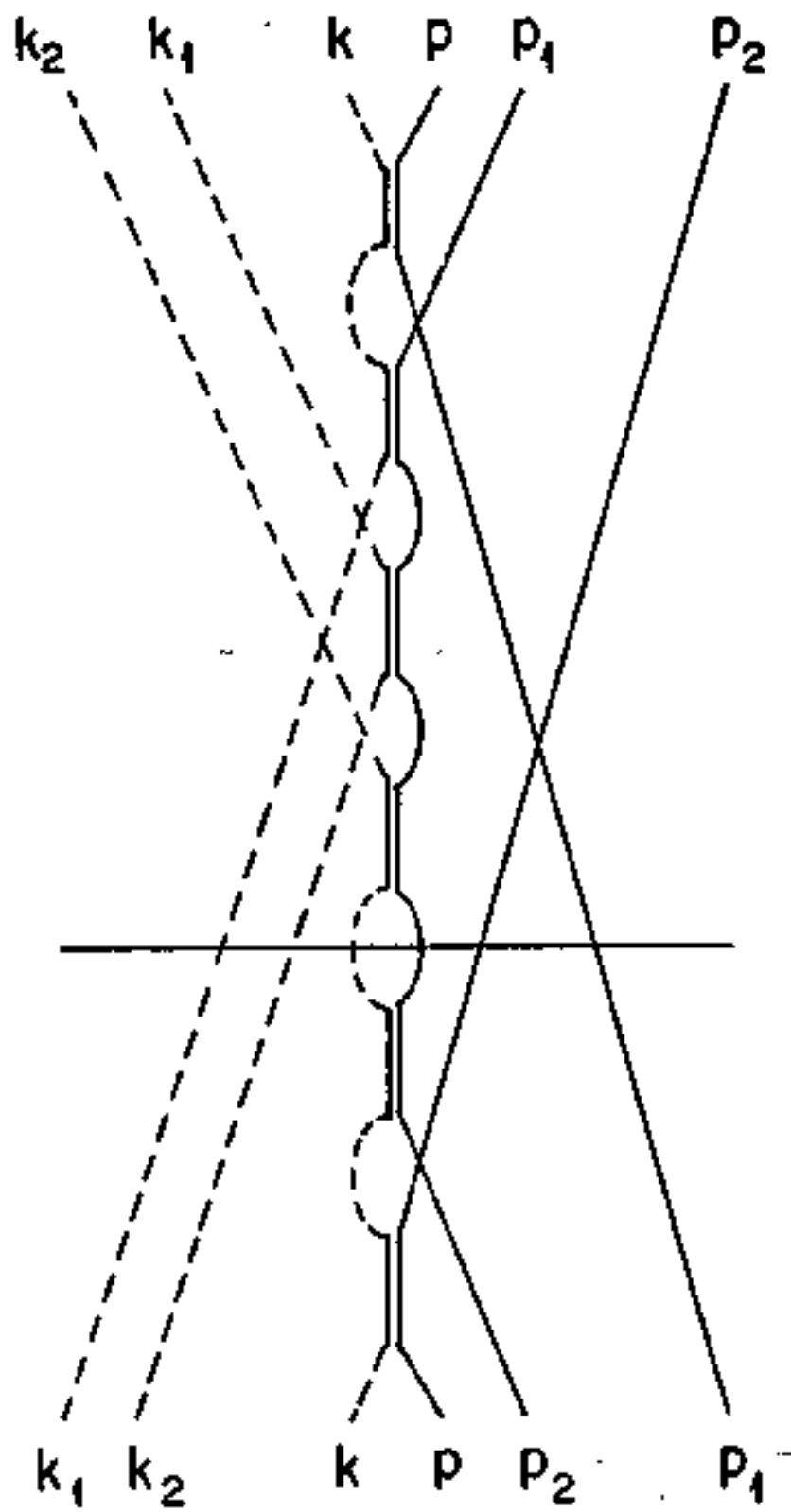

FIGURE 10 


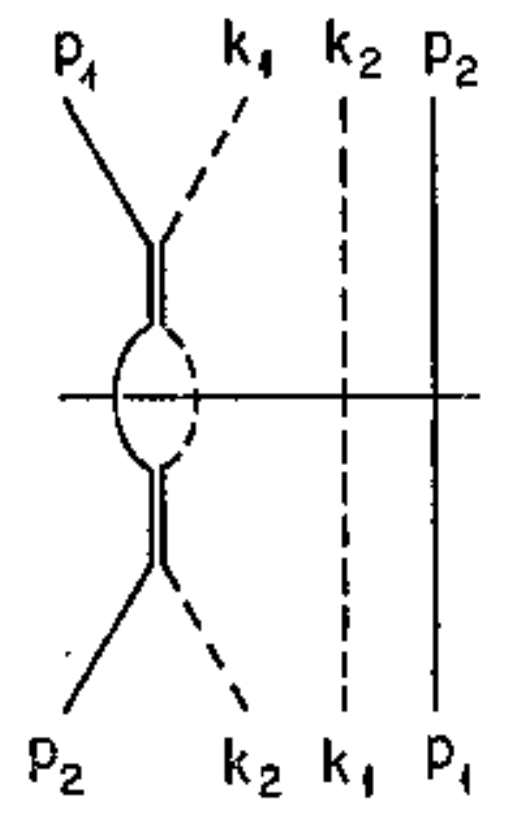

(a)

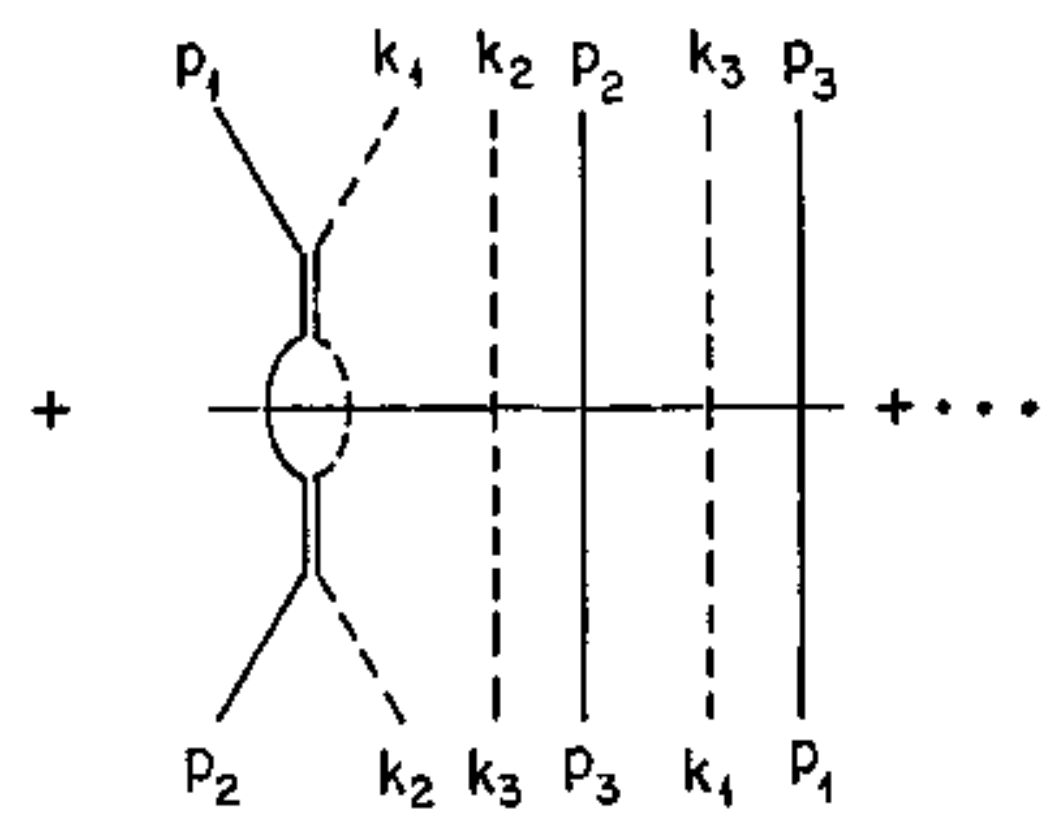

(b)

FIGURE 1 । 


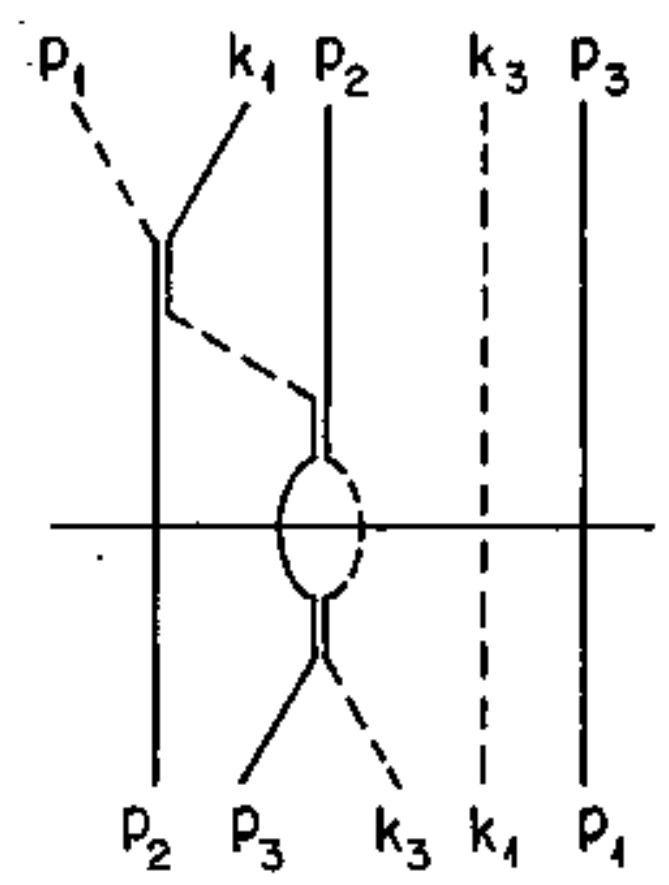

(a)

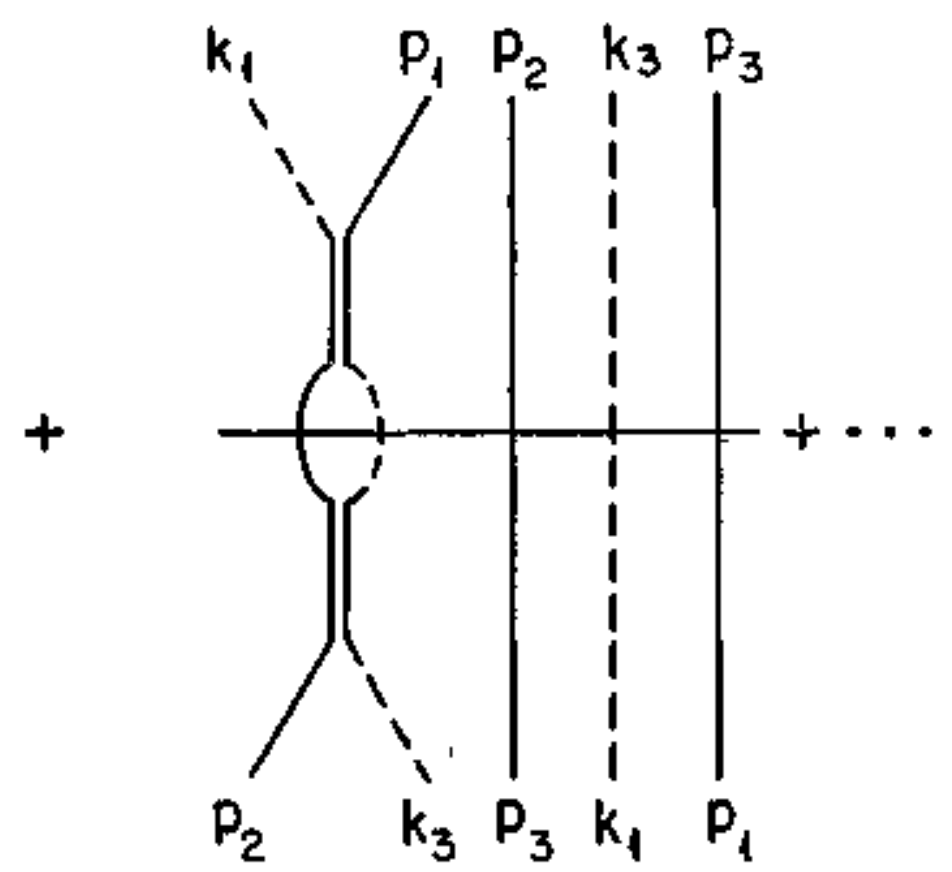

(b)

FIGURE I

i 


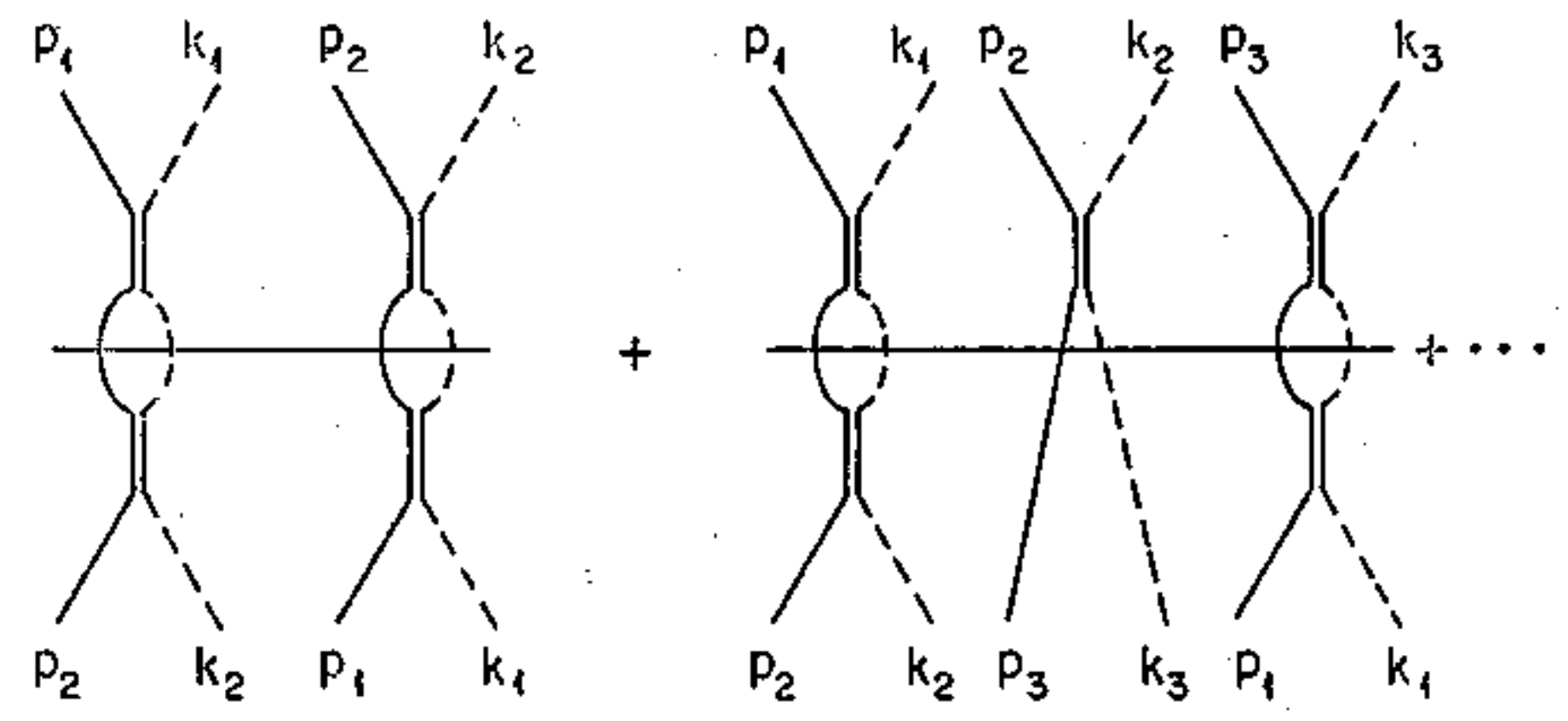

(a)

(b)

FIGURE 13 
i

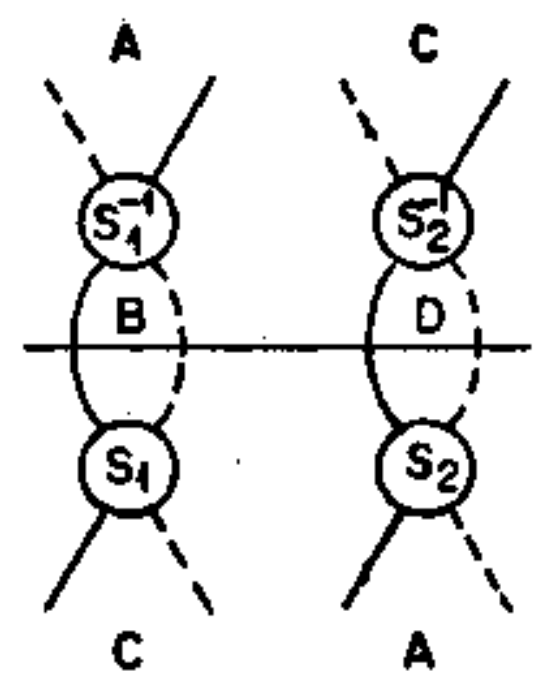

FIGURE 14 


$$
\text { X }
$$




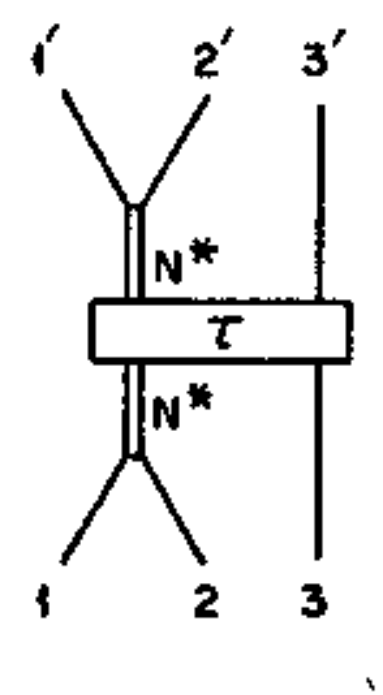

(a)

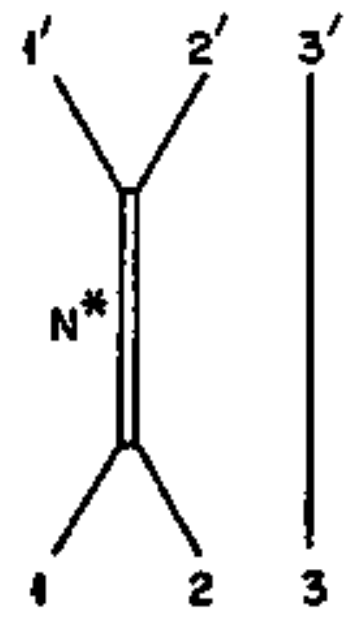

(b)

FIGURE 16 


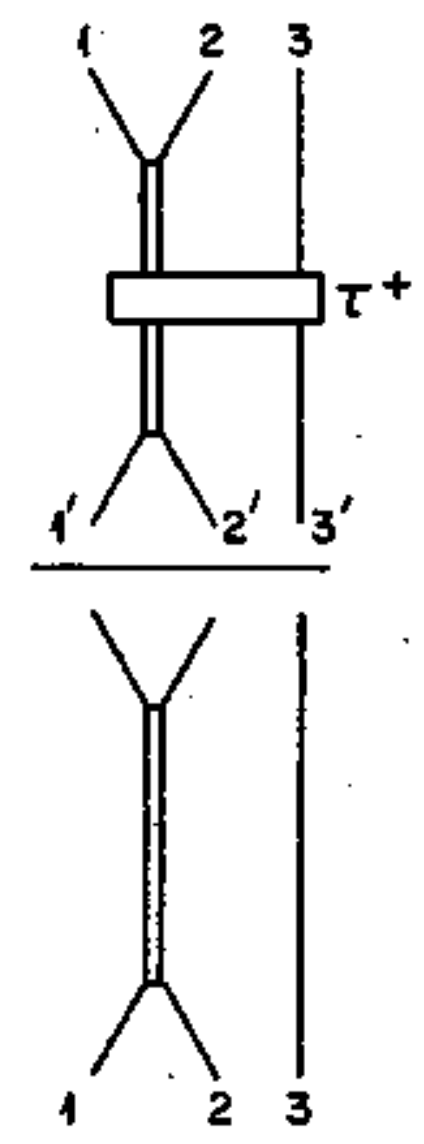

(a)

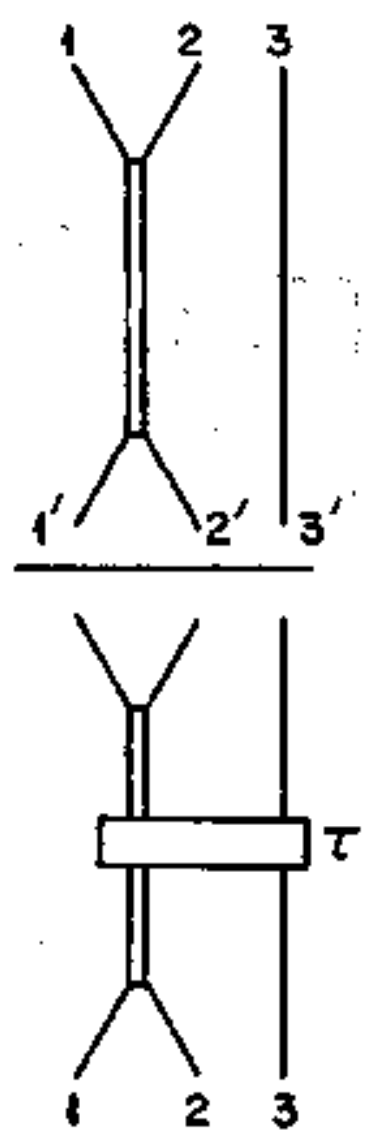

(b)

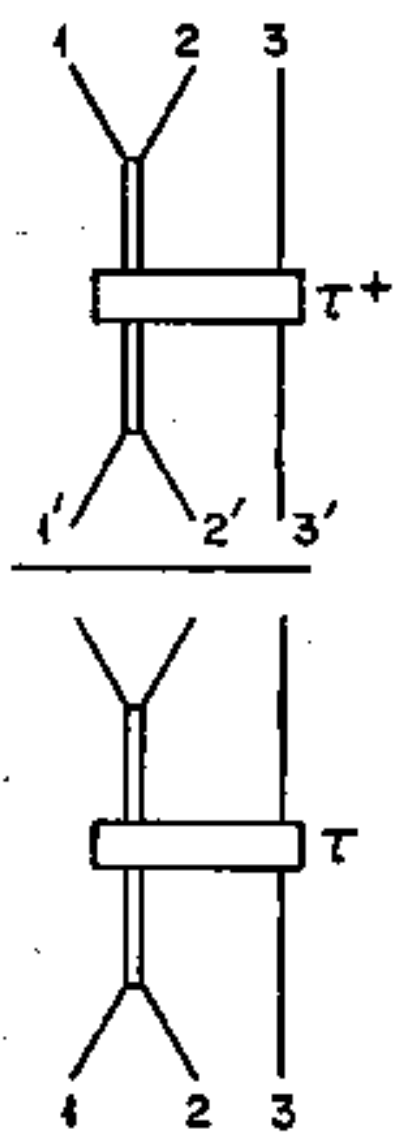

(c)

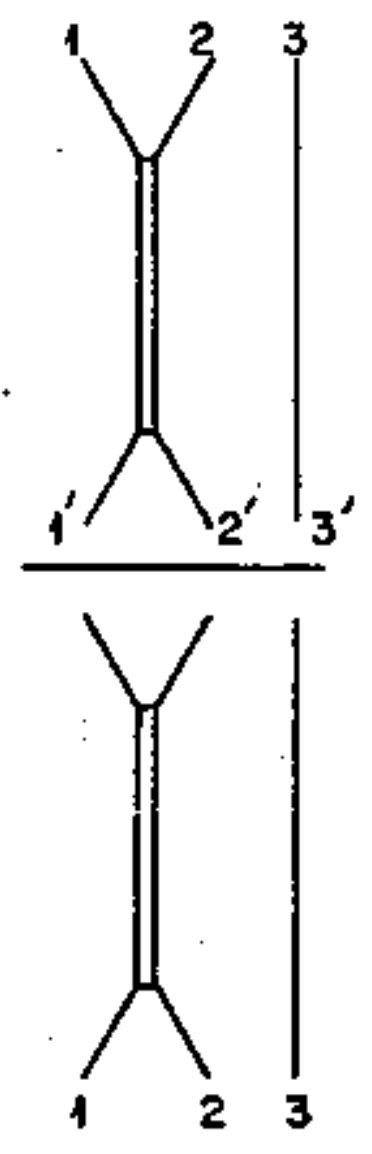

(d)

FIGURE 17 


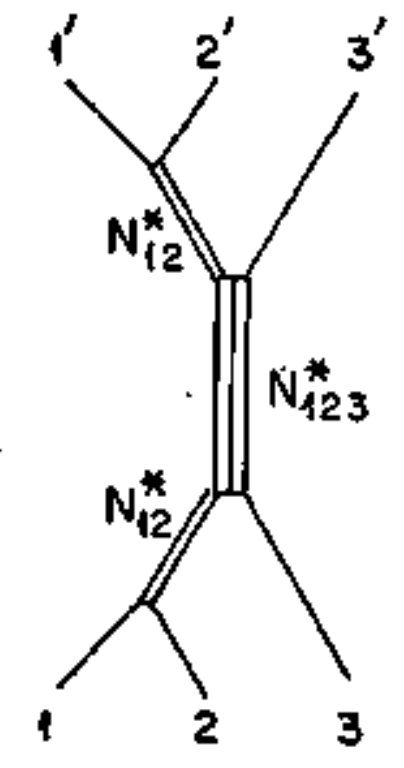

FIGURE 18 